Proceedings of the Edinburgh Mathematical Society (2005) 48, 603-629 (C)

DOI:10.1017/S0013091504000148 Printed in the United Kingdom

\title{
MICROLOCAL ANALYSIS OF GENERALIZED FUNCTIONS: PSEUDODIFFERENTIAL TECHNIQUES AND PROPAGATION OF SINGULARITIES
}

\author{
CLAUDIA GARETTO ${ }^{1}$ AND GÜNTHER HÖRMANN ${ }^{2}$ \\ ${ }^{1}$ Dipartimento di Matematica, Università di Torino, \\ Palazzo Campana Via Carlo Alberto 10, 10123 Torino, \\ Italy (garettoc@dm.unito.it) \\ ${ }^{2}$ Fakultät für Mathematik, Universität Wien, Nordbergstraße 15, \\ 1090 Wien, Austria (guenther.hoermann@univie.ac.at)
}

(Received 20 February 2004)

\begin{abstract}
We characterize microlocal regularity, in the $\mathcal{G}^{\infty}$-sense, of Colombeau generalized functions by an appropriate extension of the classical notion of micro-ellipticity to pseudodifferential operators with slow-scale generalized symbols. Thus we obtain an alternative, yet equivalent, way of determining generalized wavefront sets that is analogous to the original definition of the wavefront set of distributions via intersections over characteristic sets. The new methods are then applied to regularity theory of generalized solutions of (pseudo)differential equations, where we extend the general non-characteristic regularity result for distributional solutions and consider propagation of $\mathcal{G}^{\infty}$-singularities for homogeneous first-order hyperbolic equations.
\end{abstract}

Keywords: algebras of generalized functions; wavefront sets; pseudodifferential operators

2000 Mathematics subject classification: Primary 46F30

Secondary 35S99; 35D10

\section{Introduction}

Microlocal analysis in Colombeau algebras of generalized functions, which was initiated (in published form) in $[\mathbf{4}, \mathbf{1 9}]$, is a compatible extension of its distribution-theoretic analogue to the realm of an unrestricted differential-algebraic context. The main emphasis in recent research on the subject has been on microlocal properties of basic nonlinear operations as well as on regularity theory for generalized solutions to partial differential and pseudodifferential equations (cf. $[6,7,12-14,16,18]$ ).

For Schwartz distributions the so-called elementary characterization of microlocal regularity is a corollary to its original definition via characteristic sets under pseudodifferential actions (see [9]), whereas the intuitively appealing function-like aspect of Colombeau generalized functions seems to have fostered a 'generalized elementary' approach as being a natural definition there. This may be for two reasons. Firstly, the new microlocal regularity notion is based on $\mathcal{G}^{\infty}$-regularity, which coincides with $\mathcal{C}^{\infty}$-regularity in case 
of embedded distributions, and was introduced in $[\mathbf{2 0}]$ in direct analytical terms in form of asymptotic estimates of the derivatives. Secondly, as soon as one puts oneself into the much wider setting of Colombeau spaces - with the possibility of allowing for highly singular symbols of (pseudo)differential operators - the question of good choices for appropriate generalized notions of the characteristic set or (micro-)ellipticity turns into a considerable and crucial part of the research issue (cf. $[\mathbf{7}, \mathbf{1 3}, \mathbf{1 5}, \mathbf{1 6}])$.

In the present paper, we succeed in proving characterizations of the generalized wavefront set of a Colombeau generalized function in terms of intersections over certain non-ellipticity domains corresponding to pseudodifferential operators yielding $\mathcal{G}^{\infty}$-regular images. Thus we obtain direct analogues of Hörmander's definition of the distributional wavefront set given in [9]. Moreover, as the first test applications of the new results, we discuss a generalization of the non-characteristic regularity theorem for pseudodifferential equations and propagation of $\mathcal{G}^{\infty}$-singularities (or rather, generalized wavefront sets) for generalized solutions of first-order hyperbolic differential equations with $\mathcal{C}^{\infty}$-coefficients.

In the remainder of the introductory section we fix some notation and review basic notions from Colombeau theory. Section 2 provides the technical background on the generalized symbol classes used later on and introduces an appropriate micro-ellipticity notion. The theoretical core of the paper is $\S 3$, where the main results on micro-locality, microsupport and the wavefront set characterizations are proven. Section 4 discusses applications to regularity theory of generalized solutions of (pseudo)differential equations. Since by now several variants of pseudodifferential operator approaches in Colombeau algebras and generalized symbol calculi occur in the literature $[\mathbf{6}, \mathbf{7}, \mathbf{1 9}]$, and we employ yet a slightly different variant here, we sketch the basics of a general scheme of calculus in the appendix, the skeleton of which is structurally close to the comprehensive treatment in [7].

We point out that [19] already includes a result on micro-locality (similar to our Theorem 3.6) of actions of generalized pseudodifferential operators, whose definition is based solely on regularizing nets of symbols, rather than Colombeau classes, and uses Fourier integral representations with additional asymptotic cut-offs. The definitions of the operator actions can thus be compared in a weak sense only.

\subsection{Notation and basic notions from Colombeau theory}

We use $[\mathbf{2}, \mathbf{3}, \mathbf{8}, \mathbf{2 0}]$ as standard references for the foundations and various applications of Colombeau theory. We will work with the so-called special Colombeau algebras, denoted by $\mathcal{G}^{\mathrm{s}}$ in [8], although here we will consistently drop the superscript 's' to avoid notational overload.

We briefly recall the basic construction. Throughout the paper $\Omega$ will denote an open subset of $\mathbb{R}^{n}$. Colombeau generalized functions on $\Omega$ are defined as equivalence classes $u=$ $\left[\left(u_{\varepsilon}\right)_{\varepsilon}\right]$ of nets of smooth functions $u_{\varepsilon} \in \mathcal{C}^{\infty}(\Omega)$ (regularizations) subjected to asymptotic norm conditions with respect to $\varepsilon \in(0,1]$ for their derivatives on compact sets. More precisely, we have the following.

(i) Moderate nets $\mathcal{E}_{M}(\Omega):\left(u_{\varepsilon}\right)_{\varepsilon} \in \mathcal{C}^{\infty}(\Omega)^{(0,1]}$ such that, for all $K \Subset \Omega$ and $\alpha \in \mathbb{N}^{n}$, there exists $p \in \mathbb{R}$ such that

$$
\left\|\partial^{\alpha} u_{\varepsilon}\right\|_{L^{\infty}(K)}=O\left(\varepsilon^{-p}\right) \quad(\varepsilon \rightarrow 0) .
$$


(ii) Negligible nets $\mathbb{N}(\Omega):\left(u_{\varepsilon}\right)_{\varepsilon} \in \mathcal{E}_{M}(\Omega)$ such that, for all $K \Subset \Omega$ and for all $q \in \mathbb{R}$, an estimate $\left\|u_{\varepsilon}\right\|_{L^{\infty}(K)}=O\left(\varepsilon^{q}\right)(\varepsilon \rightarrow 0)$ holds.

(iii) $\mathcal{E}_{M}(\Omega)$ is a differential algebra with operations defined at fixed $\varepsilon, \mathbb{N}(\Omega)$ is an ideal, and $\mathcal{G}(\Omega):=\mathcal{E}_{M}(\Omega) / \mathbb{N}(\Omega)$ is the (special) Colombeau algebra.

(iv) There are embeddings, $\mathcal{C}^{\infty}(\Omega) \hookrightarrow \mathcal{G}(\Omega)$ as subalgebra and $\mathcal{D}^{\prime}(\Omega) \hookrightarrow \mathcal{G}(\Omega)$ as linear space, commuting with partial derivatives.

(v) $\Omega \rightarrow \mathcal{G}(\Omega)$ is a fine sheaf and $\mathcal{G}_{\mathrm{c}}(\Omega)$ denotes the subalgebra of elements with compact support; using a cut-off in a neighbourhood of the support, one can always obtain representing nets with supports contained in a joint compact set.

The subalgebra $\mathcal{G}^{\infty}(\Omega)$ of regular Colombeau, or $\mathcal{G}^{\infty}$-regular, generalized functions consists of those elements in $\mathcal{G}(\Omega)$ possessing representatives such that estimate (1.1) holds for a certain $m$ uniformly over all $\alpha \in \mathbb{N}^{n}$. We will occasionally use the notation $\mathcal{E}_{M}^{\infty}(\Omega)$ for the set of such nets of regularizations. In a similar sense, we will denote by $\mathcal{E}_{\mathcal{S}}^{\infty}\left(\mathbb{R}^{n}\right)$ the set of regularizations $\left(v_{\varepsilon}\right)_{\varepsilon} \in \mathcal{S}\left(\mathbb{R}^{n}\right)^{(0,1]}$ with a uniform asymptotic power of $\varepsilon$-growth for all $\mathcal{S}$-seminorms of $v_{\varepsilon}$.

A Colombeau generalized function $u=\left[\left(u_{\varepsilon}\right)_{\varepsilon}\right] \in \mathcal{G}(\Omega)$ is said to be generalized microlocally regular, or $\mathcal{G}^{\infty}$-microlocally regular, at $\left(x_{0}, \xi_{0}\right) \in T^{*}(\Omega) \backslash 0=\Omega \times\left(\mathbb{R}^{n} \backslash\{0\}\right)$ (cotangent bundle with the zero section removed) if there is $\phi \in \mathcal{C}_{\mathrm{c}}^{\infty}(\Omega)$ with $\phi\left(x_{0}\right)=1$ and a conic neighbourhood $\Gamma \subseteq \mathbb{R}^{n} \backslash\{0\}$ of $\xi_{0}$ such that $\mathcal{F}(\phi u)$ is (Colombeau) rapidly decreasing in $\Gamma$ (cf. [11]), i.e. there exists $N$ such that, for all $l$, we have

$$
\sup _{\xi \in \Gamma}\langle\xi\rangle^{l}\left|\widehat{\left(\phi u_{\varepsilon}\right)}(\xi)\right|=O\left(\varepsilon^{-N}\right), \quad \varepsilon \rightarrow 0,
$$

where we have used the standard notation $\langle\xi\rangle=\left(1+|\xi|^{2}\right)^{1 / 2}$. Note that, instead of specifying the test function $\phi$ as above, one may equivalently require the existence of an open neighbourhood $U$ of $x_{0}$ such that, for all $\phi \in \mathcal{C}_{\mathrm{c}}^{\infty}(U)$, the estimate (1.2) holds.

Finally, we will use the term proper cut-off function for any $\chi \in \mathcal{C}^{\infty}(\Omega \times \Omega)$ such that $\operatorname{supp}(\chi)$ is a proper subset of $\Omega \times \Omega$ (i.e. both projections are proper maps) and $\chi=1$ in a neighbourhood of the diagonal $\{(x, x): x \in \Omega\} \subset \Omega \times \Omega$.

\section{Slow-scale micro-ellipticity}

The pseudodifferential operator techniques which we employ are based on a generalization of the classical symbol spaces $S^{m}\left(\Omega \times \mathbb{R}^{n}\right)$ (cf. [9]). These spaces are Fréchet spaces endowed with the seminorms

$$
|a|_{K, \alpha, \beta}^{(m)}=\sup _{x \in K, \xi \in \mathbb{R}^{n}}\langle\xi\rangle^{-m+|\alpha|}\left|\partial_{\xi}^{\alpha} \partial_{x}^{\beta} a(x, \xi)\right|,
$$

where $K$ ranges over the compact subsets of $\Omega$. Several types of Colombeau generalized symbols are studied in $[\mathbf{6}, \mathbf{7}, \mathbf{1 2}, \mathbf{1 9}]$, providing a pseudodifferential calculus and regularity theory on the level of operators. In the current paper, we focus on the microlocal aspects. 
To this end we introduce a particularly flexible class of symbols with good stability properties with respect to lower-order perturbations. In the spirit of the earlier Colombeau approaches, our symbols are defined via families $\left(a_{\varepsilon}\right)_{\varepsilon} \in S^{m}\left(\Omega \times \mathbb{R}^{n}\right)^{(0,1]}=: \mathcal{S}^{m}\left[\Omega \times \mathbb{R}^{n}\right]$ of regularizations, subjected to asymptotic estimates of the above seminorms in terms of $\varepsilon$. The particular new feature is a slow-scale growth, which proved to be essential in regularity theory $(\mathrm{cf} .[\mathbf{7}, \mathbf{1 5}, \mathbf{1 6}])$. This property is measured by the elements of the following set of strongly positive slow-scale nets:

$$
\Pi_{\mathrm{sc}}:=\left\{\left(\omega_{\varepsilon}\right)_{\varepsilon} \in \mathbb{R}^{(0,1]}: \exists c>0 \forall \varepsilon: c \leqslant \omega_{\varepsilon}, \forall p \geqslant 0 \exists c_{p}>0 \forall \varepsilon: \omega_{\varepsilon}^{p} \leqslant c_{p} \varepsilon^{-1}\right\} .
$$

Definition 2.1. Let $m$ be a real number. The set of slow-scale nets of symbols of order $m$ is defined by

$$
\begin{aligned}
\mathcal{S}_{\mathrm{sc}}^{m}\left(\Omega \times \mathbb{R}^{n}\right):=\left\{\left(a_{\varepsilon}\right)_{\varepsilon} \in \mathcal{S}^{m}\left[\Omega \times \mathbb{R}^{n}\right]:\right. & \forall K \Subset \Omega \exists\left(\omega_{\varepsilon}\right)_{\varepsilon} \in \Pi_{\mathrm{sc}}, \\
& \left.\forall \alpha, \beta \in \mathbb{N}^{n} \exists c>0 \forall \varepsilon:\left|a_{\varepsilon}\right|_{K, \alpha, \beta}^{(m)} \leqslant c \omega_{\varepsilon}\right\},
\end{aligned}
$$

the negligible nets of symbols of order $m$ are the elements of

$$
\begin{aligned}
& \underline{\mathcal{N}}^{m}\left(\Omega \times \mathbb{R}^{n}\right):=\left\{\left(a_{\varepsilon}\right)_{\varepsilon} \in \mathcal{S}^{m}\left[\Omega \times \mathbb{R}^{n}\right]: \forall K \Subset \Omega \forall \alpha, \beta \in \mathbb{N}^{n},\right. \\
&\left.\forall q \in \mathbb{N}, \exists c>0 \forall \varepsilon:\left|a_{\varepsilon}\right|_{K, \alpha, \beta}^{(m)} \leqslant c \varepsilon^{q}\right\} .
\end{aligned}
$$

The classes of the factor space

$$
\underline{\mathcal{S}}_{\mathrm{sc}}^{m}\left(\Omega \times \mathbb{R}^{n}\right):=\underline{\mathcal{S}}_{\mathrm{sc}}^{m}\left(\Omega \times \mathbb{R}^{n}\right) / \underline{\mathcal{N}}^{m}\left(\Omega \times \mathbb{R}^{n}\right)
$$

are called slow-scale generalized symbols of order $m$.

Furthermore, let

$$
\underline{\mathcal{N}}^{-\infty}\left(\Omega \times \mathbb{R}^{n}\right):=\bigcap_{m} \underline{\mathcal{N}}^{m}\left(\Omega \times \mathbb{R}^{n}\right)
$$

be the negligible nets of order $-\infty$. The slow-scale generalized symbols of refined order $m$ are given by

$$
\tilde{\mathcal{S}}_{\mathrm{sc}}^{m /-\infty}\left(\Omega \times \mathbb{R}^{n}\right):=\underline{\mathcal{S}}_{\mathrm{sc}}^{m}\left(\Omega \times \mathbb{R}^{n}\right) / \underline{\mathcal{N}}^{-\infty}\left(\Omega \times \mathbb{R}^{n}\right) .
$$

Note that $\tilde{\mathcal{S}}_{\mathrm{sc}}^{m /-\infty}\left(\Omega \times \mathbb{R}^{n}\right)$ can be viewed as a finer partitioning of the classes in $\tilde{\mathcal{S}}_{\mathrm{sc}}^{m}\left(\Omega \times \mathbb{R}^{n}\right)$; in other words, if $a$ is a slow-scale generalized symbol of order $m$, then $\forall\left(b_{\varepsilon}\right)_{\varepsilon} \in a:$

$$
\kappa\left(\left(b_{\varepsilon}\right)_{\varepsilon}\right):=\left(b_{\varepsilon}\right)_{\varepsilon}+\underline{\mathcal{N}}^{-\infty}\left(\Omega \times \mathbb{R}^{n}\right) \subseteq\left(b_{\varepsilon}\right)_{\varepsilon}+\underline{\mathcal{N}}^{m}\left(\Omega \times \mathbb{R}^{n}\right)=a .
$$

Slow-scale generalized symbols enable us to design a particularly simple, yet sufficiently strong, notion of micro-ellipticity.

Definition 2.2. Let $a \in \tilde{\mathcal{S}}_{\mathrm{sc}}^{m}\left(\Omega \times \mathbb{R}^{n}\right)$ and $\left(x_{0}, \xi_{0}\right) \in T^{*}(\Omega) \backslash 0$. We say that $a$ is slowscale micro-elliptic at $\left(x_{0}, \xi_{0}\right)$ if it has a representative $\left(a_{\varepsilon}\right)_{\varepsilon}$ satisfying the following condition: there is a relatively compact open neighbourhood $U$ of $x_{0}$, a conic neighbourhood $\Gamma$ of $\xi_{0}$, and $\left(r_{\varepsilon}\right)_{\varepsilon},\left(s_{\varepsilon}\right)_{\varepsilon} \in \Pi_{\mathrm{sc}}$ such that

$$
\left|a_{\varepsilon}(x, \xi)\right| \geqslant \frac{1}{s_{\varepsilon}}\langle\xi\rangle^{m}, \quad(x, \xi) \in U \times \Gamma,|\xi| \geqslant r_{\varepsilon}, \varepsilon \in(0,1] .
$$


We denote by $\operatorname{Ell}_{\mathrm{sc}}(a)$ the set of all $\left(x_{0}, \xi_{0}\right) \in T^{*}(\Omega) \backslash 0$ where $a$ is slow-scale micro-elliptic.

If there exists $\left(a_{\varepsilon}\right)_{\varepsilon} \in a$ such that $(2.6)$ holds at all points in $T^{*}(\Omega) \backslash 0$, then the symbol $a$ is called slow-scale elliptic.

Note that here the use of the attribute 'slow scale' refers to the appearance of the slowscale lower bound in (2.6). This is a crucial difference from more general definitions of ellipticity given in $[\mathbf{6}, \mathbf{7}, \mathbf{1 6}]$, whereas a similar condition has already been used in $[\mathbf{1 5}, \S 6]$ in a special case. In fact, due to the overall slow-scale conditions in Definition 2.1, any symbol which is slow-scale micro-elliptic at $\left(x_{0}, \xi_{0}\right)$ fulfils the stronger hypoellipticity estimates [7, Definition 6.1]; furthermore, (2.6) is stable under lower-order (slow-scale) perturbations.

Proposition 2.3. Let $\left(a_{\varepsilon}\right)_{\varepsilon} \in \underline{\mathcal{S}}_{\mathrm{sc}}^{m}\left(\Omega \times \mathbb{R}^{n}\right)$ satisfy (2.6) in $U \times \Gamma \ni\left(x_{0}, \xi_{0}\right)$. Then

(i) for all $\alpha, \beta \in \mathbb{N}^{n}$ there exists $\left(\lambda_{\varepsilon}\right)_{\varepsilon} \in \Pi_{\mathrm{sc}}$ such that

$$
\left|\partial_{\xi}^{\alpha} \partial_{x}^{\beta} a_{\varepsilon}(x, \xi)\right| \leqslant \lambda_{\varepsilon}\left|a_{\varepsilon}(x, \xi)\right|\langle\xi\rangle^{-|\alpha|}, \quad(x, \xi) \in U \times \Gamma,|\xi| \geqslant r_{\varepsilon}, \varepsilon \in(0,1] ;
$$

(ii) for all $\left(b_{\varepsilon}\right)_{\varepsilon} \in \underline{S}_{\mathrm{sc}}^{m^{\prime}}\left(\Omega \times \mathbb{R}^{n}\right), m^{\prime}<m$, there exist $\left(r_{\varepsilon}^{\prime}\right)_{\varepsilon},\left(s_{\varepsilon}^{\prime}\right)_{\varepsilon} \in \Pi_{\mathrm{sc}}$ such that

$$
\left|a_{\varepsilon}(x, \xi)+b_{\varepsilon}(x, \xi)\right| \geqslant \frac{1}{s_{\varepsilon}^{\prime}}\langle\xi\rangle^{m}, \quad(x, \xi) \in U \times \Gamma,|\xi| \geqslant r_{\varepsilon}^{\prime}, \varepsilon \in(0,1] .
$$

Proof. Combining (2.6) with the seminorm estimates of $\left(a_{\varepsilon}\right)_{\varepsilon} \in \underline{S}_{\mathrm{sc}}^{m}\left(\Omega \times \mathbb{R}^{n}\right)$, we find that, for $(x, \xi) \in U \times \Gamma,|\xi| \geqslant r_{\varepsilon}, \varepsilon \in(0,1]$,

$$
\left|\partial_{\xi}^{\alpha} \partial_{x}^{\beta} a_{\varepsilon}(x, \xi)\right| \leqslant c \omega_{\varepsilon}\langle\xi\rangle^{m-|\alpha|} \leqslant c \omega_{\varepsilon} s_{\varepsilon}\left|a_{\varepsilon}(x, \xi)\right|\langle\xi\rangle^{-|\alpha|},
$$

so that assertion (i) holds with $\lambda_{\varepsilon}=c \omega_{\varepsilon} s_{\varepsilon}$. To prove (ii), again by (2.6) for $\left(a_{\varepsilon}\right)_{\varepsilon}$ and the seminorm estimates for $\left(b_{\varepsilon}\right)_{\varepsilon}$ we obtain

$$
\left|a_{\varepsilon}(x, \xi)+b_{\varepsilon}(x, \xi)\right| \geqslant \frac{1}{s_{\varepsilon}}\langle\xi\rangle^{m}-c \omega_{\varepsilon}\langle\xi\rangle^{m^{\prime}}=\langle\xi\rangle^{m}\left(\frac{1}{s_{\varepsilon}}-c \omega_{\varepsilon}\langle\xi\rangle^{m^{\prime}-m}\right),
$$

which is bounded from below by $\langle\xi\rangle^{m} / 2 s_{\varepsilon}$ whenever $(x, \xi) \in U \times \Gamma$ with

$$
|\xi| \geqslant \max \left(r_{\varepsilon},\left(2 c \omega_{\varepsilon} s_{\varepsilon}\right)^{1 /\left(m-m^{\prime}\right)}\right) .
$$

\section{Remark 2.4.}

(i) In the case of classical symbols, the notion of slow-scale micro-ellipticity coincides with the classical one, which equivalently is defined as the set of non-characteristic points. Indeed, if $a \in S^{m}\left(\Omega \times \mathbb{R}^{n}\right)$ and $\left(a_{\varepsilon}\right)_{\varepsilon}$ is a representative of the class of $a$ in $\tilde{\mathcal{S}}_{\mathrm{sc}}^{m}\left(\Omega \times \mathbb{R}^{n}\right)$ satisfying $(2.6)$ then, for any $q \in \mathbb{N}$,

$$
|a(x, \xi)| \geqslant\left|a_{\varepsilon}(x, \xi)\right|-\left|\left(a-a_{\varepsilon}\right)(x, \xi)\right| \geqslant\langle\xi\rangle^{m}\left(\frac{1}{s_{\varepsilon}}-c \varepsilon^{q}\right),
$$

where we are free to fix $\varepsilon$ small enough such that the last factor is bounded away from 0. In particular, we find that $\operatorname{Ell}_{\mathrm{sc}}(a)^{\mathrm{c}}=\operatorname{Char}(a(x, D))$. 
(ii) The same Definition 2.2 can be applied to symbols of refined order. In that case, by Proposition $2.3,(2.6)$ will hold for any representative once it is known to hold for one. Moreover, if $a \in \tilde{\mathcal{S}}_{\mathrm{sc}}^{m /-\infty}\left(\Omega \times \mathbb{R}^{n}\right)$ and $\operatorname{Ell}_{\mathrm{sc}}(a)^{\mathrm{c}}=\emptyset$, then $a$ is slow-scale elliptic.

Thanks to the previous proposition, the simple slow-scale ellipticity condition in Definition 2.2 already guarantees the existence of a parametrix. For the proof we refer to $[\mathbf{7}, \S 6]$; note that an inspection of the construction shows that the symbol of the parametrix has uniform growth $\varepsilon^{-1}$ over all compact sets. Regular symbols are introduced in Remark A 2 .

Theorem 2.5. Let a be a slow-scale elliptic symbol of order $m$. Then there exists a properly supported pseudodifferential operator with regular symbol $p \in \tilde{\mathcal{S}}_{\mathrm{rg}}^{-m}\left(\Omega \times \mathbb{R}^{n}\right)$ such that, for all $u \in \mathcal{G}_{\mathrm{c}}(\Omega)$,

$$
\begin{aligned}
& a(x, D) \circ p(x, D) u=u+R u, \\
& p(x, D) \circ a(x, D) u=u+S u,
\end{aligned}
$$

where $R$ and $S$ are operators with regular kernel.

Note that, if $a(x, D)$ is properly supported, then the operators $R$ and $S$ are properly supported too, and the previous equalities are valid for all $u$ in $\mathcal{G}(\Omega)$. In this situation, combining the construction of a parametrix with the pseudolocality property (see the appendix for details), we find that $\operatorname{singsupp}_{g}(a(x, D) u)=\operatorname{singsupp}_{g}(u)$ for all $u \in \mathcal{G}(\Omega)$.

In the following, ${ }_{\mathrm{pr}} \Psi_{\mathrm{sc}}^{m}(\Omega)$ denotes the set of all properly supported operators $a(x, D)$, where $a$ belongs to $\tilde{\mathcal{S}}_{\mathrm{sc}}^{m}\left(\Omega \times \mathbb{R}^{n}\right)$. We are now in a position to introduce a way to measure the regularity of Colombeau generalized functions mimicking the original definition of the distributional wavefront set in [9] based on characteristic sets. As a matter of fact, the set constructed below as the complement of the slow-scale micro-ellipticity regions will turn out to be the generalized wavefront set in the sense of $[\mathbf{4}, \mathbf{1 1}]$.

Definition 2.6. Let $u \in \mathcal{G}(\Omega)$. We define

$$
W_{\mathrm{sc}}(u):=\bigcap_{\substack{a(x, D) \in \in_{\mathrm{pr}} \Psi_{\mathrm{sc}}^{0}(\Omega) \\ a(x, D) u \in \mathcal{G}^{\infty}(\Omega)}} \operatorname{Ell}_{\mathrm{sc}}(a)^{\mathrm{c}} .
$$

Remark 2.7. Note that the standard procedure of lifting symbol orders with $(1-\Delta)^{m / 2}$ easily shows that we may as well take the intersection over operators $a(x, D) \in{ }_{\mathrm{pr}} \Psi_{\mathrm{sc}}^{m}(\Omega)$ in $(2.7)$. The same holds for similar constructions introduced throughout the paper.

Since $\operatorname{Ell}_{\mathrm{sc}}(a)^{\mathrm{c}}$ is a closed conic set, $W_{\mathrm{sc}}(u)$ is a closed conic subset of $T^{*}(\Omega) \backslash 0$ as well. Moreover, recalling that, given $v \in \mathcal{G}^{\infty}(\Omega)$ and $a(x, D)$ properly supported, $a(x, D)(u+v) \in \mathcal{G}^{\infty}(\Omega)$ if and only if $a(x, D) u \in \mathcal{G}^{\infty}(\Omega)$, we have $W_{\mathrm{sc}}(u+v)=W_{\mathrm{sc}}(u)$.

We present now our first alternative way of defining $W_{\mathrm{sc}}(u)$, which will be useful in the course of our exposition. Denote by ${ }_{\mathrm{pr}} \Psi_{\mathrm{sc}}^{m /-\infty}(\Omega)$ the set of all properly supported 
operators $a(x, D)$, where $a \in \tilde{\mathcal{S}}_{\mathrm{sc}}^{m /-\infty}\left(\Omega \times \mathbb{R}^{n}\right)$; one can prove that

$$
W_{\mathrm{sc}}(u)=\bigcap_{\substack{a(x, D) \in \in_{\mathrm{pr}} \Psi_{\mathrm{sc}}^{0 /-\infty}(\Omega) \\ a(x, D) u \in \mathcal{G}^{\infty}(\Omega)}} \operatorname{Ell}_{\mathrm{sc}}(a)^{\mathrm{c}} .
$$

In fact, the crucial point is to observe that we do not change a pseudodifferential operator with generalized symbol $a$ by adding negligible nets of symbols of the same order or of order $-\infty$. To be more precise, if $a(x, D) \in{ }_{\mathrm{pr}} \Psi_{\mathrm{sc}}^{0}(\Omega),\left(x_{0}, \xi_{0}\right) \in \operatorname{Ell}_{\mathrm{sc}}(a)$ with $\left(a_{\varepsilon}\right)_{\varepsilon}$ satisfying (2.6), then

$$
b(x, \xi):=\left(a_{\varepsilon}\right)_{\varepsilon}+\underline{\mathcal{N}}^{-\infty}\left(\Omega \times \mathbb{R}^{n}\right)
$$

is slow-scale micro-elliptic at $\left(x_{0}, \xi_{0}\right), b(x, D) \in{ }_{\mathrm{pr}} \Psi_{\mathrm{sc}}^{0 /-\infty}(\Omega)$, since $b(x, D) \equiv a(x, D)$ and consequently we have the inclusion $\supseteq$ in $(2.8)$. For the reverse inclusion if $a(x, D) \in$ ${ }_{\mathrm{pr}} \Psi_{\mathrm{sc}}^{0 /-\infty}(\Omega)$ with $\left(x_{0}, \xi_{0}\right) \in \operatorname{Ell}_{\mathrm{sc}}(a)$, it is clear that $b=\left(a_{\varepsilon}\right)_{\varepsilon}+\underline{\mathcal{N}}^{0}\left(\Omega \times \mathbb{R}^{n}\right)$ is a welldefined element of $\tilde{\mathcal{S}}_{\text {sc }}^{0}\left(\Omega \times \mathbb{R}^{n}\right)$ and slow-scale elliptic at $\left(x_{0}, \xi_{0}\right)$. Arguing as before, we obtain (2.8). From a technical point of view, the most interesting aspect of (2.8) is the stability of micro-ellipticity estimates under variations of the representatives of $a$, valid for symbols of refined order due to Proposition 2.3.

Proposition 2.8. Let $\pi: T^{*}(\Omega) \backslash 0 \rightarrow \Omega:(x, \xi) \rightarrow x$. For any $u \in \mathcal{G}(\Omega)$,

$$
\pi\left(W_{\mathrm{sc}}(u)\right)=\operatorname{singsupp}_{\mathrm{g}}(u) .
$$

Proof. We first prove that $\Omega \backslash \operatorname{singsupp}_{\mathrm{g}}(u) \subseteq \Omega \backslash \pi\left(W_{\mathrm{sc}}(u)\right)$. Let

$$
x_{0} \in \Omega \backslash \operatorname{singsupp}_{\mathrm{g}}(u) .
$$

There exists $\phi \in \mathcal{C}_{\mathrm{c}}^{\infty}(\Omega)$ such that $\phi\left(x_{0}\right)=1$ and $\phi u \in \mathcal{G}^{\infty}(\Omega)$. The multiplication operator $\phi(x, D): \mathcal{G}_{\mathrm{c}}(\Omega) \rightarrow \mathcal{G}_{\mathrm{c}}(\Omega): u \rightarrow \phi u$ is properly supported with symbol

$$
\phi \in S^{0}\left(\Omega \times \mathbb{R}^{n}\right) \subseteq \tilde{\mathcal{S}}_{\mathrm{sc}}^{0}\left(\Omega \times \mathbb{R}^{n}\right),
$$

which is micro-elliptic (slow-scale micro-elliptic) at $\left(x_{0}, \xi_{0}\right)$ for each $\xi_{0} \neq 0$. Therefore, for all $\xi_{0} \neq 0$, we have that $\left(x_{0}, \xi_{0}\right) \in W_{\mathrm{sc}}(u)^{\mathrm{c}}$, i.e. $x_{0} \in \Omega \backslash \pi\left(W_{\mathrm{sc}}(u)\right)$.

To show the opposite inclusion, let $x_{0} \in \Omega \backslash \pi\left(W_{\mathrm{sc}}(u)\right)$. Then for all $\xi \neq 0$ there exists

$$
a \in \tilde{\mathcal{S}}_{\mathrm{sc}}^{0 /-\infty}\left(\Omega \times \mathbb{R}^{n}\right)
$$

slow-scale micro-elliptic at $\left(x_{0}, \xi\right)$ such that $a(x, D)$ is properly supported and $a(x, D) u \in$ $\mathcal{G}^{\infty}(\Omega)$. Since $S_{x_{0}}:=\left\{x_{0}\right\} \times\{\xi:|\xi|=1\}$ is a compact subset of $\Omega \times \mathbb{R}^{n}$, there exist $a_{1}, \ldots, a_{N} \in \tilde{\mathcal{S}}_{\mathrm{sc}}^{0 /-\infty}\left(\Omega \times \mathbb{R}^{n}\right), U$ a relatively compact open neighbourhood of $x_{0}$ and $\Gamma_{i}$ conic neighbourhoods of $\xi_{i}$ with $\left|\xi_{i}\right|=1(i=1, \ldots, N)$, with the following properties: $a_{i}$ is slow-scale micro-elliptic in $U \times \Gamma_{i}, S_{x_{0}} \subseteq U \times \bigcup_{i=1}^{N} \Gamma_{i}, a_{i}(x, D)$ is properly supported, and $a_{i}(x, D) u \in \mathcal{G}^{\infty}(\Omega)$. Consider the properly supported pseudodifferential operator $A:=\sum_{i=1}^{N} a_{i}(x, D)^{*} a_{i}(x, D)$. By Theorem A 12 in the appendix we may write

$$
A=\sigma(x, D) \in{ }_{\mathrm{pr}} \Psi_{\mathrm{sc}}^{0 /-\infty}(\Omega),
$$


and combining assertions (ii) and (iii) of the same theorem, we have that

$$
\sigma-\sum_{i=1}^{N}\left|a_{i}\right|^{2} \in \tilde{\mathcal{S}}_{\mathrm{sc}}^{-1 /-\infty}\left(\Omega \times \mathbb{R}^{n}\right) .
$$

Since $a_{i}(x, D) u \in \mathcal{G}^{\infty}(\Omega)$ and each $a_{i}(x, D)^{*}$ maps $\mathcal{G}^{\infty}(\Omega)$ into $\mathcal{G}^{\infty}(\Omega)$, we conclude that $\sigma(x, D) u \in \mathcal{G}^{\infty}(\Omega)$.

It is clear that $\sum_{i=1}^{N}\left|a_{i}\right|^{2}$ is slow-scale elliptic in $U$. In fact, every $\xi \neq 0$ belongs to some $\Gamma_{i}$ and, given $\left(s_{i, \varepsilon}\right)_{\varepsilon},\left(r_{i, \varepsilon}\right)_{\varepsilon}$ satisfying $(2.6)$ for $\left(a_{i, \varepsilon}\right)_{\varepsilon}, s_{\varepsilon}:=\max _{i}\left(s_{i, \varepsilon}^{2}\right), r_{\varepsilon}:=$ $\max \left(r_{i, \varepsilon}\right)$, we get

$$
\sum_{i=1}^{N}\left|a_{i, \varepsilon}(x, \xi)\right|^{2} \geqslant \frac{1}{s_{\varepsilon}} \quad x \in U,|\xi| \geqslant r_{\varepsilon}, \varepsilon \in(0,1] .
$$

Let $U^{\prime} \subset U^{\prime \prime} \subset U$ be open neighbourhoods of $x_{0}, \overline{U^{\prime}} \subset U^{\prime \prime}, \overline{U^{\prime \prime}} \subset U$ and $\phi \in \mathcal{C}^{\infty}(\Omega)$, $0 \leqslant \phi \leqslant 1$ such that $\phi=0$ on $U^{\prime}$ and $\phi=1$ on $\Omega \backslash U^{\prime \prime}$. By construction

$$
b(x, \xi):=\phi(x)+\sigma(x, \xi) \in \tilde{\mathcal{S}}_{\mathrm{sc}}^{0 /-\infty}\left(\Omega \times \mathbb{R}^{n}\right), \quad b(x, D) \in{ }_{\mathrm{pr}} \Psi_{\mathrm{sc}}^{0 /-\infty}(\Omega)
$$

and

$$
\left.b(x, D) u\right|_{U^{\prime}}=\left.\phi u\right|_{U^{\prime}}+\left.\sigma(x, D) u\right|_{U^{\prime}}=\left.\sigma(x, D) u\right|_{U^{\prime}} \in \mathcal{G}^{\infty}\left(U^{\prime}\right) .
$$

Since $\sum_{i=1}^{N}\left|a_{i}\right|^{2}$ is slow-scale elliptic in $U$, with a positive real-valued representative, and $\phi$ is identically 1 outside $U^{\prime \prime}$, we have that $\phi+\sum_{i=1}^{N}\left|a_{i}\right|^{2}$ is slow-scale elliptic in $\Omega$. By (2.9), and application of Proposition 2.3(ii), $b$ itself is slow-scale elliptic in $\Omega$. Then, by using a parametrix for $b(x, D)$ we conclude that $\operatorname{singsupp}_{g}(b(x, D) u)=\operatorname{singsupp}_{g}(u)$ and consequently $U^{\prime} \cap \operatorname{singsupp}_{g}(u)=\emptyset$, which completes the proof.

\section{Pseudodifferential characterization of the generalized wavefront set}

This section is devoted to the proof that $W_{\mathrm{sc}}(u)$ coincides with the generalized wavefront set of $u$. Our approach will follow the lines of reasoning in [5, Chapter 8] and [10, $\S 18.1]$. The main tool will be a generalization of the micro-support of a regular generalized symbol of order $m$ or refined order $m$.

Definition 3.1. Let $a \in \tilde{\mathcal{S}}_{\mathrm{rg}}^{m}\left(\Omega \times \mathbb{R}^{n}\right)$ and $\left(x_{0}, \xi_{0}\right) \in T^{*}(\Omega) \backslash 0$. The symbol $a$ is $\mathcal{G}^{\infty}$-smoothing at $\left(x_{0}, \xi_{0}\right)$ if there exist a representative $\left(a_{\varepsilon}\right)_{\varepsilon}$ of $a$, a relatively compact open neighbourhood $U$ of $x_{0}$, a conic neighbourhood $\Gamma$ of $\xi_{0}$, and a natural number $N$ such that

$$
\forall m \in \mathbb{R} \forall \alpha, \beta \in \mathbb{N}^{n} \exists c>0 \forall(x, \xi) \in U \times \Gamma \forall \varepsilon \in(0,1]:\left|\partial_{\xi}^{\alpha} \partial_{x}^{\beta} a_{\varepsilon}(x, \xi)\right| \leqslant c\langle\xi\rangle^{m} \varepsilon^{-N} .
$$

We define the generalized microsupport of $a$, denoted by $\mu \operatorname{supp}_{g}(a)$, as the complement of the set of points $\left(x_{0}, \xi_{0}\right)$ where $a$ is $\mathcal{G}^{\infty}$-smoothing.

If $a \in \tilde{\mathcal{S}}_{\mathrm{rg}}^{m /-\infty}\left(\Omega \times \mathbb{R}^{n}\right)$, then we denote by $\mu_{\mathrm{g}}(a)$ the complement of the set points $\left(x_{0}, \xi_{0}\right) \in T^{*}(\Omega) \backslash 0$, where (3.1) holds for some representative of $a$. 


\section{Remark 3.2.}

(i) Any $a \in \tilde{\mathcal{S}}_{\mathrm{rg}}^{-\infty}\left(\Omega \times \mathbb{R}^{n}\right)$, i.e. a regular generalized symbol of order $-\infty$, has empty generalized microsupport.

(ii) In the case of $a \in \underline{\mathcal{S}}_{\mathrm{rg}}^{m /-\infty}\left(\Omega \times \mathbb{R}^{n}\right)$, every representative is of the form $\left(a_{\varepsilon}\right)_{\varepsilon}+\left(n_{\varepsilon}\right)_{\varepsilon}$, where $\left(a_{\varepsilon}\right)_{\varepsilon} \in \underline{\mathcal{S}}_{\mathrm{rg}}^{m}\left(\Omega \times \mathbb{R}^{n}\right)$ and $\left(n_{\varepsilon}\right)_{\varepsilon} \in \underline{\mathcal{N}}^{-\infty}\left(\Omega \times \mathbb{R}^{n}\right)$, and (3.1) holds for any representative once it is known to hold for one. As a consequence, if $a$ is a classical symbol of order $m$ considered as an element of $\tilde{\mathcal{S}}_{\mathrm{rg}}^{m /-\infty}\left(\Omega \times \mathbb{R}^{n}\right)$, then its generalized microsupport $\mu_{\mathrm{g}}(a)$ equals the classical one.

(iii) If $a \in \underline{\mathcal{S}}_{\mathrm{rg}}^{m /-\infty}\left(\Omega \times \mathbb{R}^{n}\right.$ and $\mu_{\mathrm{g}}(a)=\emptyset$, then $a \in \tilde{\mathcal{S}}_{\mathrm{rg}}^{-\infty}\left(\Omega \times \mathbb{R}^{n}\right)$. In fact, having chosen any $\left(a_{\varepsilon}\right)_{\varepsilon} \in a$, for all $x_{0} \in \Omega$, the compact set $S_{x_{0}}$ can be covered by $U \times \bigcup_{i=1}^{N} \Gamma_{i}$, with $U$ and $\Gamma_{i}$ such that (3.1) is valid. Hence, there exists $N \in \mathbb{N}$ such that for all orders $m \in \mathbb{R}$ and, for all $\alpha$ and $\beta$,

$$
\left|\partial_{\xi}^{\alpha} \partial_{x}^{\beta} a_{\varepsilon}(x, \xi)\right| \leqslant c\langle\xi\rangle^{m-|\alpha|} \varepsilon^{-N}, \quad(x, \xi) \in U \times \mathbb{R}^{n}, \varepsilon \in(0,1] .
$$

(iv) If $a \in \underline{\mathcal{S}}_{\mathrm{rg}}^{m}\left(\Omega \times \mathbb{R}^{n}\right)$ and $\kappa$ is the quotient map $\underline{\mathcal{S}}_{\mathrm{rg}}^{m}\left(\Omega \times \mathbb{R}^{n}\right)$ onto $\tilde{\mathcal{S}}_{\mathrm{rg}}^{m /-\infty}\left(\Omega \times \mathbb{R}^{n}\right)$, then

$$
\mu \operatorname{supp}_{\mathrm{g}}(a)=\bigcap_{\left(a_{\varepsilon}\right)_{\varepsilon} \in a} \mu_{\mathrm{g}}\left(\kappa\left(\left(a_{\varepsilon}\right)_{\varepsilon}\right)\right) .
$$

Observe first that, for all $\left(a_{\varepsilon}\right)_{\varepsilon} \in a$, we have $\mu \operatorname{supp}_{g}(a) \subseteq \mu_{\mathrm{g}}\left(\kappa\left(\left(a_{\varepsilon}\right)_{\varepsilon}\right)\right)$. On the other hand, if $\left(x_{0}, \xi_{0}\right) \notin \mu \operatorname{supp}_{g}(a)$, then $\left(x_{0}, \xi_{0}\right) \notin \mu_{\mathrm{g}}\left(\kappa\left(\left(a_{\varepsilon}\right)_{\varepsilon}\right)\right)$ for some $\left(a_{\varepsilon}\right)_{\varepsilon} \in a$.

Similarly, as in the previous section, we introduce the notation

$$
{ }_{\mathrm{pr}} \Psi_{\mathrm{rg}}^{m}(\Omega) \text { and }{ }_{\mathrm{pr}} \Psi_{\mathrm{rg}}^{m /-\infty}(\Omega)
$$

for the sets of all properly supported operators $a(x, D)$ with a symbol in

$$
\underline{\mathcal{S}}_{\mathrm{rg}}^{m}\left(\Omega \times \mathbb{R}^{n}\right) \quad \text { and } \quad \underline{\mathcal{S}}_{\mathrm{rg}}^{m /-\infty}\left(\Omega \times \mathbb{R}^{n}\right),
$$

respectively.

Proposition 3.3. Let

$$
a(x, D) \in{ }_{\mathrm{pr}} \Psi_{\mathrm{rg}}^{m /-\infty}(\Omega) \quad \text { and } \quad b(x, D) \in{ }_{\mathrm{pr}} \Psi_{\mathrm{rg}}^{m^{\prime} /-\infty}(\Omega) .
$$

Then, there exists a symbol $a \sharp b \in \tilde{\mathcal{S}}_{\mathrm{rg}}^{m+m^{\prime} /-\infty}\left(\Omega \times \mathbb{R}^{n}\right)$ such that $a(x, D) \circ b(x, D)=$ $a \sharp b(x, D) \in{ }_{\mathrm{pr}} \Psi_{\mathrm{rg}}^{m+m^{\prime} /-\infty}(\Omega)$ and

$$
\mu_{\mathrm{g}}(a \sharp b) \subseteq \mu_{\mathrm{g}}(a) \cap \mu_{\mathrm{g}}(b) .
$$

In the same situation, without regarding refined orders, we find that

$$
\mu \operatorname{supp}_{g}(a \sharp b) \subseteq \mu \operatorname{supp}_{g}(a) \cap \mu \operatorname{supp}_{g}(b) .
$$


Proof. Theorem A 12 provides the existence of $a \sharp b \in \underline{\mathcal{S}}_{\mathrm{rg}}^{m+m^{\prime} /-\infty}\left(\Omega \times \mathbb{R}^{n}\right)$ such that

$$
a(x, D) \circ b(x, D)=a \sharp b(x, D) \in{ }_{\mathrm{pr}} \Psi_{\mathrm{rg}}^{m+m^{\prime} /-\infty}(\Omega)
$$

with asymptotic expansion $a \sharp b \sim \sum_{\gamma} \partial_{\xi}^{\gamma} a D_{x}^{\gamma} b / \gamma$ !. Assume that $\left(x_{0}, \xi_{0}\right) \notin \mu_{\mathrm{g}}(a)$. By (3.1) and the symbol properties of $\left(b_{\varepsilon}\right)_{\varepsilon} \in b$, we obtain the following estimate, valid on some neighbourhood $U \times \Gamma$ of $\left(x_{0}, \xi_{0}\right)$ :

$$
\exists N \in \mathbb{N} \forall m \in \mathbb{R}, \forall \alpha, \beta \in \mathbb{N}^{n}, \quad\left|\partial_{\xi}^{\alpha} \partial_{x}^{\beta}\left(\partial_{\xi}^{\gamma} a_{\varepsilon} D_{x}^{\gamma} b_{\varepsilon}\right)(x, \xi)\right| \leqslant c\langle\xi\rangle^{m} \varepsilon^{-N} .
$$

Since $a \sharp b \sim \sum_{\gamma} \partial_{\xi}^{\gamma} a D_{x}^{\gamma} b / \gamma$ !, we find that, for any $\left(d_{\varepsilon}\right)_{\varepsilon} \in a \sharp b$ and $h \geqslant 1$, the difference

$$
\left(d_{\varepsilon}-\sum_{|\gamma| \leqslant h-1} \frac{1}{\gamma !} \partial_{\xi}^{\gamma} a_{\varepsilon} D_{x}^{\gamma} b_{\varepsilon}\right)_{\varepsilon}
$$

is an element of $\mathcal{S}_{\mathrm{rg}}^{m+m^{\prime}-h}\left(\Omega \times \mathbb{R}^{n}\right)$ and of growth type $\varepsilon^{-M}$ on $\bar{U}$, for some $M \in \mathbb{N}$ independent of $h$. This, together with (3.5), implies that $\left(x_{0}, \xi_{0}\right)$ does not belong to $\mu_{\mathrm{g}}(a \sharp b)$. The proof of relation (3.4) is similar.

We recall a technical lemma proved in [5, Proposition (8.52)] which will be useful below.

Lemma 3.4. Suppose $\left(x_{0}, \xi_{0}\right) \in T^{*}(\Omega) \backslash 0, U$ is a relatively compact open neighbourhood of $x_{0}, \Gamma$ is a conic neighbourhood of $\xi_{0}$. There exists $p \in S^{0}\left(\Omega \times \mathbb{R}^{n}\right)$ such that $0 \leqslant p \leqslant 1, \operatorname{supp}(p) \subseteq U \times \Gamma$ and $p(x, \xi)=1$ if $(x, \xi) \in U^{\prime} \times \Gamma^{\prime}$ and $|\xi| \geqslant 1$, where $U^{\prime} \times \Gamma^{\prime}$ is a smaller conic neighbourhood of $\left(x_{0}, \xi_{0}\right)$. In particular, $p$ is micro-elliptic at $\left(x_{0}, \xi_{0}\right)$ and $\mu \operatorname{supp}(p) \subseteq U \times \Gamma$.

Remark 3.5. The proof of the above lemma shows that, for each conic neighbourhood $\Gamma$ of $\xi_{0}$, there exists $\tau(\xi) \in S^{0}\left(\Omega \times \mathbb{R}^{n}\right)$ such that $0 \leqslant \tau \leqslant 1, \operatorname{supp}(\tau) \subseteq \Gamma$ and $\tau(\xi)=1$ in some conic neighbourhood $\Gamma^{\prime}$ of $\xi_{0}$ when $|\xi| \geqslant 1$.

Note that, after multiplying $p(x, \xi)$ in Lemma 3.4 by a proper cut-off function, we obtain a properly supported operator $q(x, D) \in \Psi^{0}(\Omega)$, whose symbol is micro-elliptic at $\left(x_{0}, \xi_{0}\right)$ and $\mu \operatorname{supp}(q)=\mu \operatorname{supp}(p)$.

Theorem 3.6. For any $a(x, D) \in{ }_{\mathrm{pr}} \Psi_{\mathrm{rg}}^{m}(\Omega)$ and $u \in \mathcal{G}(\Omega)$,

$$
W_{\mathrm{sc}}(a(x, D) u) \subseteq W_{\mathrm{sc}}(u) \cap \mu \operatorname{supp}_{\mathrm{g}}(a) .
$$

Similarly, if $a(x, D) \in{ }_{\mathrm{pr}} \Psi_{\mathrm{rg}}^{m /-\infty}(\Omega)$, then

$$
W_{\mathrm{sc}}(a(x, D) u) \subseteq W_{\mathrm{sc}}(u) \cap \mu_{\mathrm{g}}(a) .
$$

Proof. We first prove the assertion (3.7) in two steps.

Step 1. $W_{\mathrm{sc}}(a(x, D) u) \subseteq \mu_{\mathrm{g}}(a)$. 
If $\left(x_{0}, \xi_{0}\right) \notin \mu_{\mathrm{g}}(a)$, then (3.1) holds on some $U \times \Gamma$, and by Lemma 3.4 we find $q \in S^{0}\left(\Omega \times \mathbb{R}^{n}\right) \subseteq \tilde{\mathcal{S}}_{\mathrm{rg}}^{0 /-\infty}\left(\Omega \times \mathbb{R}^{n}\right)$, which is micro-elliptic at $\left(x_{0}, \xi_{0}\right)$ with $\mu \operatorname{supp}(q) \subseteq$ $U \times \Gamma$. Applying Proposition 3.3, we obtain

$$
q(x, D) a(x, D)=q \sharp a(x, D) \in{ }_{\mathrm{pr}} \Psi_{\mathrm{rg}}^{m /-\infty}(\Omega)
$$

and

$$
\mu_{\mathrm{g}}(q \sharp a) \subseteq \mu_{\mathrm{g}}(q) \cap \mu_{\mathrm{g}}(a) \subseteq(U \times \Gamma) \cap \mu_{\mathrm{g}}(a)=\emptyset .
$$

Remark 3.2 (iii) shows that $q \sharp a \in \tilde{\mathcal{S}}_{\mathrm{rg}}^{-\infty}\left(\Omega \times \mathbb{R}^{n}\right)$ and therefore $q(x, D) a(x, D)$ is a properly supported pseudodifferential operator with regular kernel. This implies that $q(x, D)(a(x, D) u) \in \mathcal{G}^{\infty}(\Omega)$ and hence $\left(x_{0}, \xi_{0}\right) \notin W_{\mathrm{sc}}(a(x, D) u)$.

Step 2. $W_{\mathrm{sc}}(a(x, D) u) \subseteq W_{\mathrm{sc}}(u)$.

Let $\left(x_{0}, \xi_{0}\right) \notin W_{\mathrm{sc}}(u)$. Then, by $(2.8)$, there exists $p(x, D) \in{ }_{\mathrm{pr}} \Psi_{\mathrm{sc}}^{0 /-\infty}(\Omega)$ such that $p$ is slow-scale micro-elliptic at $\left(x_{0}, \xi_{0}\right)$ and $p(x, D) u \in \mathcal{G}^{\infty}(\Omega)$.

Claim. There exists

$$
r(x, D) \in{ }_{\mathrm{pr}} \Psi_{\mathrm{sc}}^{0 /-\infty}(\Omega)
$$

such that $r$ is micro-elliptic at $\left(x_{0}, \xi_{0}\right)$, and there exists $s(x, D) \in{ }_{\mathrm{pr}} \Psi_{\mathrm{rg}}^{m /-\infty}(\Omega)$ such that $r(x, D) a(x, D) u-s(x, D) p(x, D)^{*} p(x, D) u$ belongs to $\mathcal{G}^{\infty}(\Omega)$.

Assuming for the moment that the claim is proved, we show that it completes the proof of the theorem. The operators $s(x, D)$ and $p(x, D)^{*}$ map $\mathcal{G}^{\infty}(\Omega)$ into itself; hence, we find that $s(x, D) p(x, D)^{*} p(x, D) u \in \mathcal{G}^{\infty}(\Omega)$. The claim implies that $r(x, D) a(x, D) u \in \mathcal{G}^{\infty}(\Omega)$ and $\left(x_{0}, \xi_{0}\right) \notin W_{\mathrm{sc}}(a(x, D) u)$, since $r$ is micro-elliptic at $\left(x_{0}, \xi_{0}\right)$.

Proof of Claim. To prove the claim, we construct a slow-scale elliptic symbol based on $p$. Let $\left(p_{\varepsilon}\right)_{\varepsilon}$ be a representative of $p$ satisfying (2.6) in a conic neighbourhood $U \times \Gamma$ of $\left(x_{0}, \xi_{0}\right)$. By Lemma 3.4 there is $\psi \in S^{0}\left(\Omega \times \mathbb{R}^{n}\right), 0 \leqslant \psi \leqslant 1$, with $\operatorname{supp}(\psi) \subseteq U \times \Gamma$ and identically 1 in a smaller conic neighbourhood $U^{\prime} \times \Gamma^{\prime}$ of $\left(x_{0}, \xi_{0}\right)$ if $|\xi| \geqslant 1$. The net $\left(1+\left|p_{\varepsilon}\right|^{2}-\psi\right)_{\varepsilon}$ belongs to $\mathcal{S}_{\mathrm{sc}}^{0}\left(\Omega \times \mathbb{R}^{n}\right)$ and, by construction of $\psi$, satisfies (2.6) at all points in $T^{*}(\Omega) \backslash 0$. The pseudodifferential calculus for slow-scale generalized symbols of refined order guarantees the existence of $\sigma \in \tilde{\mathcal{S}}_{\text {sc }}^{0 /-\infty}\left(\Omega \times \mathbb{R}^{n}\right)$ such that

$$
p(x, D)^{*} p(x, D)=\sigma(x, D) \in{ }_{\mathrm{pr}} \Psi_{\mathrm{sc}}^{0 /-\infty}(\Omega) \quad \text { and } \quad \sigma-|p|^{2} \in \tilde{\mathcal{S}}_{\mathrm{sc}}^{-1 /-\infty}\left(\Omega \times \mathbb{R}^{n}\right) .
$$

Application of Proposition 2.3(ii) to this situation yields the fact that the symbol

$$
1+\sigma-\psi \in \tilde{\mathcal{S}}_{\mathrm{sc}}^{0 /-\infty}\left(\Omega \times \mathbb{R}^{n}\right)
$$

is slow-scale elliptic and coincides with $\sigma$ in a conic neighbourhood $U^{\prime} \times \Gamma^{\prime}$ of $\left(x_{0}, \xi_{0}\right)$ for $|\xi| \geqslant 1$.

Take a proper cut-off function $\chi$ and define a pseudodifferential operator via the slowscale amplitude $\chi(x, y)(1+\sigma(x, \xi)-\psi(x, \xi))$. By Theorem A 12 , this can be written in the form $b(x, D) \in{ }_{\mathrm{pr}} \Psi_{\mathrm{sc}}^{0 /-\infty}(\Omega)$, where $b-(1+\sigma-\psi) \in \tilde{\mathcal{S}}_{\mathrm{sc}}^{-\infty}\left(\Omega \times \mathbb{R}^{n}\right)$. In other words, 
$b$ itself is slow-scale elliptic and $\mu_{\mathrm{g}}(b)=\mu_{\mathrm{g}}(1+\sigma-\psi)$. In particular, since $\sigma$ and $1+$ $\sigma-\psi$ coincide on $U^{\prime} \times \Gamma^{\prime},|\xi| \geqslant 1$, we find that $\mu_{\mathrm{g}}(b-\sigma) \cap\left(U^{\prime} \times \Gamma^{\prime}\right)=\emptyset$. Theorem 2.5 gives a parametrix $t(x, D) \in{ }_{\mathrm{pr}} \Psi_{\mathrm{rg}}^{0 /-\infty}(\Omega)$ for $b(x, D)$, i.e. the operators $b(x, D) t(x, D)-I$ and $t(x, D) b(x, D)-I$ have a regular kernel. Let $r(x, D) \in{ }_{\mathrm{pr}} \Psi_{\mathrm{sc}}^{0 /-\infty}(\Omega)$ be an operator constructed as in Lemma 3.4, with classical symbol $r$, micro-elliptic at $\left(x_{0}, \xi_{0}\right)$, and $\mu \operatorname{supp}(r) \subseteq U^{\prime} \times \Gamma^{\prime}$.

We show that $r(x, D)$ and

$$
s(x, D):=r \sharp(a \sharp t)(x, D)=r(x, D) a(x, D) t(x, D) \in{ }_{\mathrm{pr}} \Psi_{\mathrm{rg}}^{m /-\infty}(\Omega)
$$

satisfy the assertions of the claim. We rewrite the difference

$$
r(x, D) a(x, D) u-s(x, D) p(x, D)^{*} p(x, D) u
$$

as

$$
r(x, D) a(x, D)(u-t(x, D) b(x, D) u)+r(x, D) a(x, D) t(x, D)(b(x, D)-\sigma(x, D)) u .
$$

Here, the first summand is in $\mathcal{G}^{\infty}(\Omega)$ due to the fact that $t(x, D)$ is a parametrix for $b(x, D)$ and the mapping properties of $r(x, D) a(x, D)$. An iterated application of Proposition 3.3 to the second summand shows that it can be written with a regular symbol of refined order $m$, having generalized micro-support contained in the region

$$
\mu \operatorname{supp}(r) \cap \mu_{\mathrm{g}}(b-\sigma) \subseteq\left(U^{\prime} \times \Gamma^{\prime}\right) \cap \mu_{\mathrm{g}}(b-\sigma)=\emptyset .
$$

Hence, it has smoothing generalized symbol and therefore the claim is proven.

Finally, we prove the assertion (3.6). Let $\left(a_{\varepsilon}\right)_{\varepsilon} \in a \in \underline{\mathcal{S}}_{\mathrm{rg}}^{m}\left(\Omega \times \mathbb{R}^{n}\right)$ and consider the corresponding symbol

$$
\kappa\left(\left(a_{\varepsilon}\right)_{\varepsilon}\right)=\left(a_{\varepsilon}\right)_{\varepsilon}+\underline{\mathcal{N}}^{-\infty}\left(\Omega \times \mathbb{R}^{n}\right) \in \underline{\mathcal{S}}_{\mathrm{rg}}^{m /-\infty}\left(\Omega \times \mathbb{R}^{n}\right) .
$$

Then $\kappa\left(\left(a_{\varepsilon}\right)_{\varepsilon}\right)(x, D) \in{ }_{\mathrm{pr}} \Psi_{\mathrm{rg}}^{m /-\infty}(\Omega)$ and $\kappa\left(\left(a_{\varepsilon}\right)\right)(x, D)=a(x, D)$. Theorem 3.6, applied to all $\left(a_{\varepsilon}\right)_{\varepsilon} \in a$, yields

$$
\bigcap_{\left(a_{\varepsilon}\right)_{\varepsilon} \in a} W_{\mathrm{sc}}\left(\kappa\left(\left(a_{\varepsilon}\right)_{\varepsilon}\right)(x, D) u\right) \subseteq W_{\mathrm{sc}}(u) \cap \bigcap_{\left(a_{\varepsilon}\right)_{\varepsilon} \in a} \mu_{\mathrm{g}}\left(\kappa\left(\left(a_{\varepsilon}\right)_{\varepsilon}\right)\right),
$$

i.e.

$$
W_{\mathrm{sc}}(a(x, D) u) \subseteq W_{\mathrm{sc}}(u) \cap \bigcap_{\left(a_{\varepsilon}\right)_{\varepsilon} \in a} \mu_{\mathrm{g}}\left(\kappa\left(\left(a_{\varepsilon}\right)_{\varepsilon}\right)\right),
$$

which completes the proof by Remark 3.2 (iv).

Corollary 3.7. Let $a(x, D) \in{ }_{\mathrm{pr}} \Psi_{\mathrm{sc}}^{m}(\Omega)$, where $a$ is a slow-scale elliptic symbol. Then for any $u \in \mathcal{G}(\Omega)$

$$
W_{\mathrm{sc}}(a(x, D) u)=W_{\mathrm{sc}}(u) .
$$


Proof. Since

$$
a \in \underline{\mathcal{S}}_{\mathrm{sc}}^{m}\left(\Omega \times \mathbb{R}^{n}\right) \subseteq \tilde{\mathcal{S}}_{\mathrm{rg}}^{m}\left(\Omega \times \mathbb{R}^{n}\right),
$$

Theorem 3.6 implies that $W_{\mathrm{sc}}(a(x, D) u) \subseteq W_{\mathrm{sc}}(u)$. Let $p(x, D)$ be a parametrix for $a(x, D)$ as in Theorem 2.5. Then $u=p(x, D) a(x, D) u+v$, where $v \in \mathcal{G}^{\infty}(\Omega)$. Therefore, $W_{\mathrm{sc}}(u)=W_{\mathrm{sc}}(p(x, D) a(x, D) u)$ and Theorem 3.6, applied to $p(x, D) \in{ }_{\mathrm{pr}} \Psi_{\mathrm{rg}}^{-m}(\Omega)$, gives $W_{\mathrm{sc}}(u) \subseteq W_{\mathrm{sc}}(a(x, D) u)$.

The statements of the above theorem and corollary are valid for pseudodifferential operators that are not necessarily properly supported, if we consider instead compactly supported generalized functions.

As in the classical theory (cf. [5]), we introduce a notion of microsupport for operators. However, in the case of generalized pseudodifferential operators we are cautious to distinguish the corresponding notions for symbols and operators by a slight change in notation, thereby taking into account the non-injectivity when mapping symbols to operators (see [7]).

Definition 3.8. Let $A$ be any properly supported pseudodifferential operator with regular symbol. We define the generalized microsupport of $A$ by

$$
\boldsymbol{\mu} \operatorname{supp}_{g}(A):=\bigcap_{\substack{a(x, D) \in \in_{\mathrm{pr}} \Psi_{\mathrm{rg}}^{m}(\Omega), a(x, D)=A}} \mu \operatorname{supp}_{g}(a) .
$$

Now we have all the technical tools at hand which enable us to identify $W_{\mathrm{sc}}(u)$ as the generalized wavefront set $\mathrm{WF}_{\mathrm{g}}(u)$.

Theorem 3.9. For all $u \in \mathcal{G}(\Omega)$,

$$
W_{\mathrm{sc}}(u)=\mathrm{WF}_{\mathrm{g}}(u) .
$$

Proof. By definition of $\mathrm{WF}_{\mathrm{g}}(u)$ the assertion which we are going to prove is the following: $\left(x_{0}, \xi_{0}\right) \notin W_{\mathrm{sc}}(u)$ if and only if there exists a representative $\left(u_{\varepsilon}\right)_{\varepsilon}$ of $u$, a cutoff function $\phi \in \mathcal{C}_{\mathrm{c}}^{\infty}(\Omega)$ with $\phi\left(x_{0}\right)=1$, a conic neighbourhood $\Gamma$ of $\xi_{0}$ and a number $N$ such that, for all $l \in \mathbb{R}$,

$$
\sup _{\xi \in \Gamma}\langle\xi\rangle^{l}\left|\widehat{\phi u_{\varepsilon}}(\xi)\right|=O\left(\varepsilon^{-N}\right) \quad \text { as } \varepsilon \rightarrow 0 .
$$

We first show sufficiency, that is if $\left(x_{0}, \xi_{0}\right) \in T^{*}(\Omega) \backslash 0$ satisfies (3.10), then it does not belong to $W_{\mathrm{sc}}(u)$. As noted in Remark 3.5, there exists $p(\xi) \in S^{0}\left(\Omega \times \mathbb{R}^{n}\right)$ with $\operatorname{supp}(p) \subseteq \Gamma$, which is identically 1 in a conical neighbourhood $\Gamma^{\prime}$ of $\xi_{0}$ when $|\xi| \geqslant 1$. We recall that on taking a typical proper cut-off $\chi$, by Theorem A11, we can write the properly supported pseudodifferential operator with amplitude $\chi(x, y) p(\xi) \phi(y)$ in the form $\sigma(x, D) \in{ }_{\mathrm{pr}} \Psi_{\mathrm{sc}}^{0 /-\infty}(\Omega)$, where $\sigma(x, \xi)-p(\xi) \phi(x) \in S^{-1}\left(\Omega \times \mathbb{R}^{n}\right)$; in particular, $\sigma(x, D) v-p(D)(\phi v) \in \mathcal{G}^{\infty}(\Omega)$ for all $v \in \mathcal{G}(\Omega)$. By assumption, $p(\xi) \phi(x)$ is micro-elliptic at $\left(x_{0}, \xi_{0}\right)$, the symbol $\sigma$ is micro-elliptic there, and from (3.10) we find that

$$
p(D) \phi(x, D) u=\left[\left(\int_{\mathbb{R}^{n}} \mathrm{e}^{\mathrm{i} x \xi} p(\xi) \widehat{\phi u_{\varepsilon}}(\xi) \mathrm{d} \xi\right)_{\varepsilon}\right] \in \mathcal{G}^{\infty}(\Omega) .
$$


Since $\sigma(x, D) u-p(D)(\phi u) \in \mathcal{G}^{\infty}(\Omega)$, we find that $\sigma(x, D) u \in \mathcal{G}^{\infty}(\Omega)$ and, hence, $\left(x_{0}, \xi_{0}\right) \notin W_{\mathrm{sc}}(u)$.

Conversely, suppose $\left(x_{0}, \xi_{0}\right) \notin W_{\text {sc }}(u)$. There is an open neighbourhood $U$ of $x_{0}$ such that $\left(x, \xi_{0}\right) \in W_{\mathrm{sc}}(u)^{\mathrm{c}}$ for all $x \in U$. Choose $\phi \in \mathcal{C}_{\mathrm{c}}^{\infty}(U)$ with $\phi\left(x_{0}\right)=1$ and define

$$
\Sigma:=\left\{\xi \in \mathbb{R}^{n} \backslash 0: \exists x \in \Omega(x, \xi) \in W_{\mathrm{sc}}(\phi u)\right\} .
$$

From Theorem 3.6 we have that $W_{\mathrm{sc}}(\phi u) \subseteq W_{\mathrm{sc}}(u) \cap\left(\operatorname{supp}(\phi) \times \mathbb{R}^{n} \backslash 0\right)$ and therefore $\xi_{0} \notin \Sigma$. Moreover, since $W_{\mathrm{sc}}(\phi u)$ is closed and conic, the $\Sigma$ itself is a closed conic subset of $\mathbb{R}^{n} \backslash 0$. Again, by Remark 3.5 there is a symbol $p(\xi) \in S^{0}\left(\Omega \times \mathbb{R}^{n}\right)$ such that $0 \leqslant p \leqslant 1, p(\xi)=1$ in a conic neighbourhood $\Gamma$ of $\xi_{0}$ when $|\xi| \geqslant 1$, and $p(\xi)=0$ in a conic neighbourhood $\Sigma_{0}$ of $\Sigma$. By construction $\mu \operatorname{supp}(p) \cap\left(\Omega \times \Sigma_{0}\right)=\emptyset$ and $W_{\mathrm{sc}}(\phi u) \subseteq \Omega \times \Sigma$. Therefore,

$$
W_{\mathrm{sc}}(p(D) \phi u) \subseteq W_{\mathrm{sc}}(\phi u) \cap \mu \operatorname{supp}(p)=\emptyset
$$

and by Proposition 2.8 we conclude that $p(D) \phi u \in \mathcal{G}^{\infty}(\Omega)$. In terms of representatives $\left(u_{\varepsilon}\right)_{\varepsilon} \in u$, this means that

$$
\left(\int_{\mathbb{R}^{n}} \mathrm{e}^{\mathrm{i} x \xi} p(\xi) \widehat{\phi u_{\varepsilon}}(\xi) \mathrm{d} \xi=\left(\phi u_{\varepsilon} * p^{\vee}\right)\right)_{\varepsilon} \in \mathcal{E}_{M}^{\infty} .
$$

Note that $p^{\vee}$ is a Schwartz function outside the origin, i.e. we find, for all $\delta>0$ and $\alpha, \beta \in \mathbb{N}^{n}$, that $\sup _{|x|>\delta}\left|x^{\alpha} \partial^{\beta} p^{\vee}(x)\right|<\infty[\mathbf{5}$, Theorem (8.8a)]. If $\operatorname{dist}(x, \operatorname{supp}(\phi))>\delta$, this yields

$$
\partial^{\alpha}\left(\phi u_{\varepsilon} * p^{\vee}\right)(x)=\int_{|y|>\delta} \phi u_{\varepsilon}(x-y) \partial^{\alpha} p^{\vee}(y) \mathrm{d} y
$$

and, for all $l>0$, the estimate

$$
\begin{aligned}
\langle x\rangle^{l}\left|\partial^{\alpha}\left(\phi u_{\varepsilon} * p^{\vee}\right)(x)\right| & \leqslant c_{1} \int_{|y|>\delta}\left|\phi u_{\varepsilon}(x-y)\right|\langle x-y\rangle^{l}\langle y\rangle^{l}\left|\partial^{\alpha} p^{\vee}(y)\right| \mathrm{d} y \\
& \leqslant c_{2} \sup _{z \in \operatorname{supp}(\phi)}\left|u_{\varepsilon}(z)\right|
\end{aligned}
$$

uniformly for such $x$. When $\delta$ is chosen to be small enough, the set of points $x$ with $\operatorname{dist}(x, \operatorname{supp}(\phi)) \leqslant \delta$ is compact in $\Omega$, and from (3.11) we find that there exists $M \in \mathbb{N}$ such that for all $l \in \mathbb{R}$,

$$
\sup \langle x\rangle^{l}\left|\partial^{\alpha}\left(\phi u_{\varepsilon} * p^{\vee}\right)(x)\right|=O\left(\varepsilon^{-M}\right) \quad \text { as } \varepsilon \rightarrow 0,
$$

where the supremum is taken over $\{x: \operatorname{dist}(x, \operatorname{supp}(\phi)) \leqslant \delta\}$. Hence, we have shown that $\left(\phi u_{\varepsilon} * p^{\vee}\right)_{\varepsilon} \in \mathcal{E}_{\mathcal{S}}^{\infty}\left(\mathbb{R}^{n}\right)$. The Fourier transform maps $\mathcal{E}_{\mathcal{S}}^{\infty}\left(\mathbb{R}^{n}\right)$ into $\mathcal{E}_{\mathcal{S}}^{\infty}\left(\mathbb{R}^{n}\right)$, which implies that

$$
\left(p(\xi) \widehat{\left(\phi u_{\varepsilon}\right)}(\xi)\right)_{\varepsilon} \in \mathcal{E}_{\delta}^{\infty}\left(\mathbb{R}^{n}\right) .
$$

Since $p(\xi)=1$ in a conic neighbourhood of $\xi_{0}$, the proof is complete.

On combing Theorem 3.6 with Theorem 3.9, the following corollary is immediate from Definition 3.8 
Corollary 3.10. For any properly supported operator $A$ with generalized regular symbol and $u \in \mathcal{G}(\Omega)$ we have

$$
\mathrm{WF}_{\mathrm{g}}(A u) \subseteq \mathrm{WF}_{\mathrm{g}}(u) \cap \boldsymbol{\mu} \operatorname{supp}_{\mathrm{g}}(A) .
$$

While the purpose of the foregoing discussion was to prepare for applications to $\mathcal{G}^{\infty}$-regularity theory for pseudodifferential equations with generalized symbols, one may, as an intermediate step, investigate the propagation of $\mathcal{G}^{\infty}$-singularities in case of differential equations with smooth coefficients. Having this special situation in mind, it is natural to consider the following set, defined for any $u \in \mathcal{G}(\Omega)$ by

$$
W_{\mathrm{cl}}(u):=\bigcap \operatorname{Char}(A),
$$

where the intersection is taken over all classical properly supported operators $A \in \Psi^{0}(\Omega)$ such that $A u \in \mathcal{G}^{\infty}(\Omega)$. A careful inspection of the proof of Theorem 3.9 suggests that $W_{\mathrm{cl}}(u)$ can be used in place of $W_{\mathrm{sc}}(u)$. Indeed, it gives an alternative characterization of $\mathrm{WF}_{\mathrm{g}}(u)$.

Theorem 3.11. For all $u \in \mathcal{G}(\Omega)$,

$$
W_{\mathrm{cl}}(u)=W_{\mathrm{sc}}(u)=\mathrm{WF}_{\mathrm{g}}(u) .
$$

Proof. The inclusion $W_{\mathrm{sc}}(u) \subseteq W_{\mathrm{cl}}(u)$ is obvious. Conversely, if $\left(x_{0}, \xi_{0}\right) \notin \mathrm{WF}_{\mathrm{g}}(u)$, as in the proof of Theorem 3.9, one can find a properly supported operator $P \in \Psi^{0}(\Omega)$ such that $\left(x_{0}, \xi_{0}\right) \notin \operatorname{Char}(P)$ and $P u \in \mathcal{G}^{\infty}(\Omega)$.

\section{Non-characteristic $\mathcal{G}^{\infty}$-regularity and propagation of singularities}

As a first application of Theorem 3.9 we prove an extension of the classical result on non-characteristic regularity for distributional solutions of arbitrary pseudodifferential equations (with smooth symbols). A generalization of this result for partial differential operators with Colombeau coefficients was achieved in [16]; here we present a version for pseudodifferential operators with slow-scale generalized symbols.

Theorem 4.1. If $P=p(x, D)$ is a properly supported pseudodifferential operator with slow-scale symbol and $u \in \mathcal{G}(\Omega)$, then

$$
\mathrm{WF}_{\mathrm{g}}(P u) \subseteq \mathrm{WF}_{\mathrm{g}}(u) \subseteq \mathrm{WF}_{\mathrm{g}}(P u) \cup \operatorname{Ell}_{\mathrm{sc}}(p)^{\mathrm{c}} .
$$

Proof. The first inclusion relation is obvious from Theorem 3.6.

Assume that $\left(x_{0}, \xi_{0}\right) \notin \mathrm{WF}_{\mathrm{g}}(P u)$ but that $p$ is micro-elliptic there. Thanks to Theorem 3.9 we can find $a(x, D) \in{ }_{\mathrm{pr}} \Psi_{\mathrm{sc}}^{0}(\Omega)$ such that $a(x, D) P u \in \mathcal{G}^{\infty}(\Omega)$ and $\left(x_{0}, \xi_{0}\right) \in$ $\mathrm{Ell}_{\mathrm{sc}}(a)$. By the (slow-scale) symbol calculus and Proposition 2.3 (ii), $a(x, D) p(x, D)$ has a slow-scale symbol, which is micro-elliptic at $\left(x_{0}, \xi_{0}\right)$. Hence, another application of Theorem 3.9 yields that $\left(x_{0}, \xi_{0}\right) \notin \mathrm{WF}_{\mathrm{g}}(u)$. 
Remark 4.2. As can be seen from various examples in [15], relation (4.1) does not hold in general for regular symbols $p$ which satisfy estimate (2.6). In this sense, the overall slow-scale properties of the symbol are crucial in the above statement and are not just technical convenience. In fact, adapting the reasoning in [15, Example 4.6] to the symbol $p_{\varepsilon}(x, \xi)=1+c_{\varepsilon} x^{2}, c_{\varepsilon} \geqslant 0$, we find that $p_{\varepsilon}(x, \xi) \geqslant 1$, whereas the unique solution $u$ of $p u=1$ is $\mathcal{G}^{\infty}$ if and only if $\left(c_{\varepsilon}\right)_{\varepsilon}$ is a slow-scale net.

In the remainder of this section we show how Theorem 3.11 enables us to extend a basic result on propagation of singularities presented in $[\mathbf{1 0}, \S 23.1]$, where we now allow for Colombeau generalized functions as solutions and initial values in first-order strictly hyperbolic partial differential equations with smooth coefficients. Hyperbolicity will be assumed with respect to time direction and we will occasionally employ pseudodifferential operators whose symbols depend smoothly on the real parameter $t$. This means that we have symbols from the space $\mathcal{C}^{\infty}\left(\mathbb{R}, S^{m}\left(\Omega \times \mathbb{R}^{n}\right)\right)$ : these are of the form $a(t, x, \xi)$ with $a \in \mathcal{C}^{\infty}\left(\mathbb{R} \times \Omega \times \mathbb{R}^{n}\right)$ such that for each $t$ and $h \in \mathbb{N}$ one has

$$
\frac{\mathrm{d}^{h}}{\mathrm{~d} t^{h}} a(t, \cdot, \cdot) \in S^{m}\left(\Omega \times \mathbb{R}^{n}\right),
$$

where all symbol seminorm estimates are uniform when $t$ varies in a compact subset of $\mathbb{R}$. Defined on representatives in the obvious way, a properly supported operator $a\left(t, x, \mathrm{D}_{x}\right)$ maps $\mathcal{G}(\Omega)$ into $\mathcal{G}(\Omega)$. Moreover, if we assume that $a\left(t, x, \mathrm{D}_{x}\right)$ is uniformly properly supported with respect to $t$, that is there exists a proper closed set $L$ such that $\operatorname{supp} k_{a\left(t, x, \mathrm{D}_{x}\right)} \subseteq L$ for all $t$, then if $u \in \mathcal{G}(\Omega \times \mathbb{R}), a\left(t, x, \mathrm{D}_{x}\right)(u(t, \cdot)) \in \mathcal{G}(\Omega \times \mathbb{R})$.

Proposition 4.3. Let $P\left(t, x, \mathrm{D}_{x}\right)$ be a first-order partial differential operator with real principal symbol $P_{1}$ and coefficients in $\mathcal{C}^{\infty}(\mathbb{R} \times \Omega)$, which are constant outside some compact subset of $\Omega$. Assume that $u \in \mathcal{G}(\Omega \times \mathbb{R})$ satisfies the homogeneous Cauchy problem

$$
\begin{aligned}
\partial_{t} u+\mathrm{i} P\left(t, x, \mathrm{D}_{x}\right) u & =0, \\
u(\cdot, 0) & =g \in \mathcal{G}(\Omega) .
\end{aligned}
$$

If $\Phi_{t}$ denotes the Hamilton flow corresponding to $P_{1}(t, \cdot, \cdot)$ on $T^{*}(\Omega)$, then we find, for all $t \in \mathbb{R}$, that

$$
\mathrm{WF}_{\mathrm{g}}(u(\cdot, t))=\Phi_{t}\left(\mathrm{WF}_{\mathrm{g}}(g)\right) .
$$

Proof. We adapt the symbol constructions presented in [10, pp. 388, 389]. Observe that one can carry out all steps of that classical procedure in $\Omega \subseteq \mathbb{R}^{n}$ and with all operators uniformly properly supported. To be more precise, let

$$
\left(x_{0}, \xi_{0}\right) \in\left(T^{*}(\Omega) \backslash 0\right) \backslash \mathrm{WF}_{\mathrm{g}}(g)
$$

and choose $q \in S^{0}\left(\Omega \times \mathbb{R}^{n}\right)$ to be polyhomogeneous, i.e. having homogeneous terms in the asymptotic expansion, such that $q$ is micro-elliptic at $\left(x_{0}, \xi_{0}\right), \mu \operatorname{supp}(q) \cap \mathrm{WF}_{\mathrm{g}}(g)=\emptyset$, and $q(x, D)$ is properly supported. By Corollary 3.10 , we deduce that $\operatorname{WF}_{\mathrm{g}}(q(x, D) g) \subseteq$ $\mathrm{WF}_{\mathrm{g}}(g) \cap \mu \operatorname{supp}(q)=\emptyset$; therefore $q(x, D) g \in \mathcal{G}^{\infty}(\Omega)$.

We can find a symbol $Q(t, x, \xi)$, which is polyhomogeneous of order 0 , smoothly depending on the parameter $t \in \mathbb{R}$, and which has the following properties: 
(i) the operators $Q\left(t, x, \mathrm{D}_{x}\right)$ are uniformly properly supported for $t \in \mathbb{R}$;

(ii) $\left[\partial_{t}+\mathrm{i} P\left(t, x, \mathrm{D}_{x}\right), Q\left(t, x, \mathrm{D}_{x}\right)\right]=R\left(t, x, \mathrm{D}_{x}\right)$ is a $t$-parametrized operator of order $-\infty$ and uniformly properly supported;

(iii) $Q_{0}(t, x, \xi)=q_{0}\left(\Phi_{t}^{-1}(x, \xi)\right)$ for the principal symbols and $Q(0, x, \xi)-q(x, \xi) \in$ $S^{-\infty}\left(\Omega \times \mathbb{R}^{n}\right)$.

From the properties of $Q$ and (4.2) we have

$$
\begin{aligned}
\left(\partial_{t}+\mathrm{i} P\left(t, x, \mathrm{D}_{x}\right)\right) Q\left(t, x, \mathrm{D}_{x}\right) u & =Q\left(t, x, \mathrm{D}_{x}\right)\left(\partial_{t} u+\mathrm{i} P\left(t, x, \mathrm{D}_{x}\right) u\right)+\left[\partial_{t}+\mathrm{i} P, Q\right] u \\
& =R\left(t, x, \mathrm{D}_{x}\right) u,
\end{aligned}
$$

and

$$
Q\left(0, x, \mathrm{D}_{x}\right) u(\cdot, 0)=q(x, D) g+R_{0}(x, D) g,
$$

where $R_{0}$ is of order $-\infty$. Observe that $R\left(t, x, \mathrm{D}_{x}\right) u(\cdot, t) \in \mathcal{G}^{\infty}(\Omega)$ and $R_{0}(x, D) g \in$ $\mathcal{G}^{\infty}(\Omega)$, which implies that $Q\left(0, x, \mathrm{D}_{x}\right) u(\cdot, 0) \in \mathcal{G}^{\infty}(\Omega)$. Setting $v=Q u \in \mathcal{G}(\Omega \times \mathbb{R})$, we obtain $\partial_{t} v+\mathrm{i} P\left(t, x, \mathrm{D}_{x}\right) v \in \mathcal{G}(\Omega \times \mathbb{R})$ and

$$
\partial_{t} v(\cdot, t)+\mathrm{i} P\left(t, x, \mathrm{D}_{x}\right) v(\cdot, t) \in \mathcal{G}^{\infty}(\Omega) \quad \forall t \in \mathbb{R}, v(\cdot, 0) \in \mathcal{G}^{\infty}(\Omega),
$$

which we interpret as a Cauchy problem with $\mathcal{G}^{\infty}$-data with respect to the space variable $x$. From an inspection of the energy estimates discussed in [17] one can infer directly that $v(\cdot, t) \in \mathcal{G}^{\infty}(\Omega)$ for all $t \in \mathbb{R}$ (note that the coefficients are independent of $\epsilon$ ). Therefore, at a fixed value of $t$ we find that $Q\left(t, x, \mathrm{D}_{x}\right) u(\cdot, t) \in \mathcal{G}^{\infty}(\Omega)$ and the noncharacteristic regularity relation (4.1) yields

$$
\begin{aligned}
\operatorname{WF}_{\mathrm{g}}(u(\cdot, t)) & \subseteq \operatorname{Char}\left(Q\left(t, x, \mathrm{D}_{x}\right)\right) \\
& =\left\{(x, \xi): Q_{0}(t, \cdot, \cdot)=q_{0}\left(\Phi_{t}^{-1}(\cdot, \cdot)\right) \text { is not micro-elliptic at }(x, \xi)\right\} .
\end{aligned}
$$

But $q_{0} \circ \Phi_{t}^{-1}$ is micro-elliptic at $(x, \xi)=\Phi_{t}\left(x_{0}, \xi_{0}\right)$, which implies that $(x, \xi) \notin$ $\mathrm{WF}_{\mathrm{g}}(u(\cdot, t))$, and therefore we have shown that

$$
\mathrm{WF}_{\mathrm{g}}(u(\cdot, t)) \subseteq \Phi_{t}\left(\mathrm{WF}_{\mathrm{g}}(g)\right) .
$$

The opposite inclusion is proved by time reversal.

Remark 4.4. The same result can be proven when $P$ is a pseudodifferential operator (with parameter $t$ and global symbol estimates), but in this case with $g$ and $u$ in the $\mathcal{G}_{2,2}$-spaces as introduced in [1] and using a basic regularity result from [12]. However, formally this requires us first to adapt the notion of micro-ellipticity to that context, which will be left to future presentations.

While Proposition 4.3 determines the wavefront set of $u(\cdot, t)$ for fixed $t$, we are aiming for a complete description of $\mathrm{WF}_{\mathrm{g}}(u)$ in the cotangent bundle over $\Omega \times \mathbb{R}$. The crucial new ingredient needed in extending the analogous discussion in [10, p. 390] to Colombeau generalized functions is a result on microlocal regularity of the restriction operator $\mathcal{G}(\Omega \times$ $\mathbb{R}) \rightarrow \mathcal{G}(\Omega)$. 
Lemma 4.5. Let $I \subseteq \mathbb{R}$ be an open interval, $t_{0} \in I$, and $u \in \mathcal{G}(\Omega \times I)$ such that

$$
\mathrm{WF}_{\mathrm{g}}(u) \cap\left(\Omega \times\left\{t_{0}\right\} \times\{(0, \tau): \tau \in \mathbb{R}\}\right)=\emptyset .
$$

Then the wavefront set of the restriction of $u$ to $\Omega \times\left\{t_{0}\right\}$ satisfies the following relation:

$$
\mathrm{WF}_{\mathrm{g}}\left(\left.u\right|_{t=t_{0}}\right) \subseteq\left\{(x, \xi) \in T^{*}(\Omega) \backslash 0: \exists \tau \in \mathbb{R}:\left(x, t_{0} ; \xi, \tau\right) \in \mathrm{WF}_{\mathrm{g}}(u)\right\} .
$$

Remark 4.6. Note that, unlike with distributions, the restriction $\left.u\right|_{t=t_{0}}$ is always well defined for Colombeau functions on $\Omega \times I$, regardless of the microlocal intersection condition (4.6) in the above lemma. However, an obvious adaption of the counter example in [14, Example 5.1] shows that, in general, (4.6) cannot be dropped without losing the relation (4.7).

Proof. We are proving a microlocal statement, so we may assume that $t_{0}=0$ and that $u$ has compact support near $\{t=0\}$; in particular, we may then pick a representative $\left(u_{\varepsilon}\right)_{\varepsilon}$ of $u$ with $\operatorname{supp}\left(u_{\varepsilon}\right)$ contained in a fixed compact set uniformly for all $\varepsilon \in(0,1]$.

The aim of the proof is to show the following: if $\left(x_{0}, \xi_{0}\right) \in T^{*}(\Omega) \backslash 0$ is in the complement of the right-hand side of relation (4.7) and $\phi \in \mathcal{C}_{\mathrm{c}}^{\infty}(\Omega)$ is supported near $x_{0}$, then $\left(\phi(\cdot) u_{\varepsilon}(\cdot, 0)\right)(\xi)$ is (Colombeau-type) rapidly decreasing, i.e. with uniform $\varepsilon$ asymptotics (see $\left[\mathbf{1 1}\right.$, Definition 17]), in some conic neighbourhood $\Gamma\left(\xi_{0}\right)$ of $\xi_{0}$. As a preliminary observation, note that if $\psi \in \mathcal{C}_{\mathrm{c}}^{\infty}(I)$ with $\psi(0)=1$, then we may write

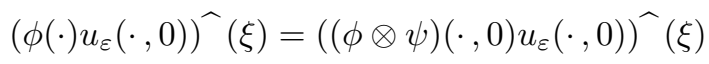

$$
\begin{aligned}
& =\int \mathcal{F}_{n+1}\left((\phi \otimes \psi) u_{\varepsilon}\right)(\xi, \tau) \mathrm{d} \tau,
\end{aligned}
$$

where $\mathcal{F}_{n+1}$ denotes $(n+1)$-dimensional Fourier transform. We will find rapid decrease estimates of the integrand upon an appropriate splitting of the integral depending on the parameter $\xi$.

First, the hypothesis (4.6) yields that, for each $y \in \Omega$, we find an open neighbourhood $V(y, 0)$ and open cones $\Gamma_{y}^{ \pm} \ni(0, \pm 1)$ such that for all $f \in \mathcal{C}_{\mathrm{c}}^{\infty}(V(y, 0))$ the function $\widehat{\left(f u_{\varepsilon}\right)}(\xi, \tau)$ is Colombeau-rapidly decreasing in $\Gamma_{y}:=\Gamma_{y}^{-} \cup \Gamma_{y}^{+}$. By compactness the set $\operatorname{supp}(u) \cap(\Omega \times\{0\})$ is covered by finitely many neighbourhoods $V\left(y_{1}, 0\right), \ldots, V\left(y_{M}, 0\right)$. Again by compactness, we may assume that there exists $U_{1} \subseteq \Omega$ open and $\eta_{1}>0$ such that

$$
\operatorname{supp}(u) \cap(\Omega \times\{0\}) \subseteq U_{1} \times\left(-\eta_{1}, \eta_{1}\right) \subseteq \bigcup_{j=1}^{M} V\left(y_{j}, 0\right) .
$$

Furthermore, $\bigcap_{j=1}^{M} \Gamma_{y_{j}}$ is an open cone around $(0,-1)$ and $(0,1)$ and there exists $c_{1}>0$ such that it still contains the conic neighbourhood $\Gamma_{1}:=\left\{(\xi, \tau):|\tau| \geqslant c_{1}|\xi|\right\}$. Using a finite partition of unity subordinated to $\left(V\left(y_{j}, 0\right)\right)_{1 \leqslant j \leqslant M}$, we obtain the following statement: for any $\phi \in \mathcal{C}_{\mathrm{c}}^{\infty}\left(U_{1}\right)$ and $\psi \in \mathcal{C}_{\mathrm{c}}^{\infty}\left(\left(-\eta_{1}, \eta_{1}\right)\right)$ there exists $N \geqslant 0$ with the property that $\forall l \in \mathbb{N} \exists C_{l}>0 \exists \varepsilon_{0}>0$ which guarantees the rapid decrease estimate

$$
\left|\mathcal{F}_{n+1}\left((\phi \otimes \psi) u_{\varepsilon}\right)(\xi, \tau)\right| \leqslant C_{l} \varepsilon^{-N}\langle(\xi, \tau)\rangle^{-l} \quad(\xi, \tau) \in \Gamma_{1}, 0<\varepsilon<\varepsilon_{0} .
$$


This will provide corresponding upper bounds for the integrand in (4.8) whenever $|\tau| \geqslant$ $c_{1}|\xi|$, for $\xi$ arbitrary.

Second, it follows from the assumption on $\left(x_{0}, \xi_{0}\right)$ that $\left(\left\{\left(x_{0}, 0\right)\right\} \times\left\{\xi_{0}\right\} \times \mathbb{R}\right) \cap \mathrm{WF}_{\mathrm{g}}(u)=$ $\emptyset$. Hence for all $\sigma \in \mathbb{R}$ there is an open set $V_{\sigma} \ni\left(x_{0}, 0\right)$ and an open conic neighbourhood $\Gamma\left(\xi_{0}, \sigma\right)$ such that, for all $f \in \mathcal{C}_{\mathrm{c}}^{\infty}\left(V_{\sigma}\right)$, the function $\widehat{\left(f u_{\varepsilon}\right)}(\xi, \tau)$ is Colombeaurapidly decreasing in $\Gamma_{\sigma}$. By a conic compactness argument (via projections to the unit sphere), we deduce that finitely many cones $\Gamma_{\sigma_{j}}(j=1, \ldots, M)$ suffice to cover the twodimensional sector $\left\{\left(\lambda \xi_{0}, \tau\right): \lambda>0,|\tau| \leqslant c_{1} \lambda\left|\xi_{0}\right|\right\}$. Let $\pi_{n}: \mathbb{R}^{n+1} \rightarrow \mathbb{R}^{n}$ be the projection $\pi_{n}(\xi, \tau)=\xi$ and define

$$
\Gamma\left(\xi_{0}\right):=\bigcap_{j=1, \ldots, M} \pi_{n}\left(\Gamma_{\sigma_{j}}\right),
$$

which is an open conic neighbourhood of $\xi_{0}$ in $\mathbb{R}^{n} \backslash 0$. Furthermore, $\bigcap_{j=1}^{M} V_{\sigma_{j}} \ni\left(x_{0}, 0\right)$ is open and contains still some neighbourhood of product form, say $U_{0} \times\left(-\eta_{0}, \eta_{0}\right)$. Therefore, for any $\phi \in \mathcal{C}_{\mathrm{c}}^{\infty}\left(U_{0}\right)$ and $\psi \in \mathcal{C}_{\mathrm{c}}^{\infty}\left(\left(-\eta_{0}, \eta_{0}\right)\right)$ there exists $N \geqslant 0$ with the following property: $\forall l \in \mathbb{N} \exists C_{l}>0 \exists \varepsilon_{0}>0$ we have an estimate

$$
\left|\mathcal{F}_{n+1}\left((\phi \otimes \psi) u_{\varepsilon}\right)(\xi, \tau)\right| \leqslant C_{l} \varepsilon^{-N}\langle(\xi, \tau)\rangle^{-l} \quad \xi \in \Gamma\left(\xi_{0}\right),|\tau| \leqslant c_{1}|\xi|,
$$

when $0<\varepsilon<\varepsilon_{0}$. So if $\xi \in \Gamma\left(\xi_{0}\right)$, we also obtain corresponding upper bounds in (4.8) over the remaining integration domain $|\tau| \leqslant c_{1}|\xi|$.

To summarize: when $\xi \in \Gamma\left(\xi_{0}\right)$ we may combine (4.9) and (4.10) by taking $U:=U_{0} \cap U_{1}$, $\phi \in \mathcal{C}_{\mathrm{c}}^{\infty}(U)$, and $\eta:=\min \left(\eta_{0}, \eta_{1}\right), \psi \in \mathcal{C}_{\mathrm{c}}^{\infty}((-\eta, \eta)), \psi(0)=1$. Upon applying this to $(4.8)$ we arrive at

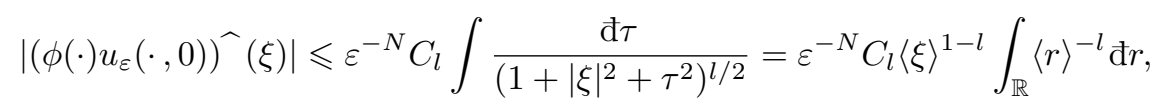

for some $N$ independent of $l \geqslant 2$ and $\varepsilon$ sufficiently small.

Theorem 4.7. Let $u$ be the (unique) solution of the homogeneous Cauchy problem (4.2), (4.3) and denote by $\gamma\left(x_{0}, \xi_{0}\right)$ the maximal bicharacteristic curve passing through the characteristic point $\left(x_{0}, 0 ; \xi_{0},-P_{1}\left(0, x_{0}, \xi_{0}\right)\right) \in T^{*}(\Omega \times \mathbb{R}) \backslash 0$. Then the generalized wavefront of $u$ is given by

$$
\mathrm{WF}_{\mathrm{g}}(u)=\bigcup_{\left(x_{0}, \xi_{0}\right) \in \mathrm{WF}_{\mathrm{g}}(g)} \gamma\left(x_{0}, \xi_{0}\right) .
$$

Proof. We refer to the discussion in [10, p. 390], which we can adapt to our case with only a few changes. First, $\partial_{t}+\mathrm{i} P\left(t, x, \mathrm{D}_{x}\right)$ is already a partial differential operator and hence we obtain $\mathrm{WF}_{\mathrm{g}}(u) \subseteq \operatorname{Char}\left(\partial_{t}+\mathrm{i} P_{1}\right) \not \supset(x, t ; 0, \tau)$ due to (4.1); therefore, combining this with Lemma 4.5, we immediately obtain the inclusion

$$
\mathrm{WF}_{\mathrm{g}}(u(\cdot, t)) \subseteq\left\{(x, \xi) \in T^{*}(\Omega) \backslash 0:\left(x, t ; \xi,-P_{1}(t, x, \xi)\right) \in \mathrm{WF}_{\mathrm{g}}(u)\right\} .
$$

On the other hand, based on the inclusion relation (4.5), we can carry out the following construction. Let $\left(x_{1}, t_{1}, \xi_{1}, \tau_{1}\right) \in T^{*}(\Omega \times \mathbb{R}) \backslash 0$, such that $\xi_{1} \neq 0$ and 
$\left(x_{1}, \xi_{1}\right) \notin \mathrm{WF}_{\mathrm{g}}\left(u\left(\cdot, t_{1}\right)\right)$, and let $Q_{0}$ be as in the proof of Proposition 4.3 , which is micro-elliptic at $\left(x_{1}, \xi_{1}\right)$. We claim that $\left(x_{1}, t_{1}, \xi_{1}, \tau_{1}\right) \notin \mathrm{WF}_{\mathrm{g}}(u)$. Indeed, one may use cut-off functions of the product form $\phi(x) \psi(t)$ with small supports near $x_{1}, t_{1}$ and write

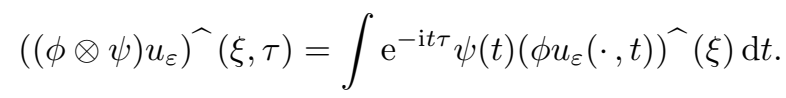

We have Colombeau rapid decrease estimates in $(\xi, \tau)$ for those $\xi$-directions where $Q(t, x, \xi)$ stays micro-elliptic for all $(x, t) \in \operatorname{supp}(\phi \otimes \psi)$. Together with the observation at the beginning of the proof and (4.4), this implies the equality

$$
\begin{aligned}
\Phi_{t}\left(\mathrm{WF}_{\mathrm{g}}(g)\right) & =\mathrm{WF}_{\mathrm{g}}(u(\cdot, t)) \\
& =\left\{(x, \xi) \in T^{*}(\Omega) \backslash 0:\left(x, t ; \xi,-P_{1}(t, x, \xi)\right) \in \mathrm{WF}_{\mathrm{g}}(u)\right\},
\end{aligned}
$$

which yields the asserted statement.

\section{Appendix A. Pseudodifferential calculus with general scales}

We provide some background to the required pseudodifferential tools for a sufficiently large class of generalized symbols.

Definition A 1. Let $\mathfrak{A}$ be the set of all nets $\left(\omega_{\varepsilon}\right)_{\varepsilon} \in \mathbb{R}^{(0,1]}$ such that $c_{0} \leqslant \omega_{\varepsilon} \leqslant c_{1} \epsilon^{-p}$ for some $c_{0}, c_{1}, p>0$ and for all $\varepsilon$. Let $\mathfrak{B}$ be any subset of $\mathfrak{A}$ closed with respect to pointwise product and maximum. For $m \in \mathbb{R}$ and $\Omega$ an open subset of $\mathbb{R}^{N}$, we define the spaces of $\mathfrak{B}$-nets of symbols:

$$
\begin{aligned}
& \mathcal{S}_{\mathfrak{B}}^{m}\left(\Omega \times \mathbb{R}^{n}\right):=\left\{\left(a_{\varepsilon}\right)_{\varepsilon} \in \mathcal{S}^{m}\left[\Omega \times \mathbb{R}^{n}\right]: \forall K \Subset \Omega \exists\left(\omega_{\varepsilon}\right)_{\varepsilon} \in \mathfrak{B},\right. \\
&\left.\forall \alpha, \beta \in \mathbb{N}^{n} \exists c>0 \forall \varepsilon:\left|a_{\varepsilon}\right|_{K, \alpha, \beta}^{(m)} \leqslant c \omega_{\varepsilon}\right\}, \\
& \mathcal{S}_{\mathfrak{B}}^{-\infty}\left(\Omega \times \mathbb{R}^{n}\right):=\left\{\left(a_{\varepsilon}\right)_{\varepsilon} \in \mathcal{S}^{m}\left[\Omega \times \mathbb{R}^{n}\right]: \forall K \Subset \Omega \exists\left(\omega_{\varepsilon}\right)_{\varepsilon} \in \mathfrak{B} \forall m \in \mathbb{R},\right. \\
&\left.\forall \alpha, \beta \in \mathbb{N}^{n} \exists c>0 \forall \varepsilon:\left|a_{\varepsilon}\right|_{K, \alpha, \beta}^{(m)} \leqslant c \omega_{\varepsilon}\right\}
\end{aligned}
$$

and the factor spaces

$$
\begin{aligned}
\tilde{\mathcal{S}}_{\mathfrak{B}}^{m}\left(\Omega \times \mathbb{R}^{n}\right) & :=\underline{\mathcal{S}}_{\mathfrak{B}}^{m}\left(\Omega \times \mathbb{R}^{n}\right) / \underline{\mathcal{N}}^{m}\left(\Omega \times \mathbb{R}^{n}\right), \\
\tilde{\mathcal{S}}_{\mathfrak{B}}^{m,-\infty}\left(\Omega \times \mathbb{R}^{n}\right) & :=\underline{\mathcal{S}}_{\mathfrak{B}}^{m}\left(\Omega \times \mathbb{R}^{n}\right) / \underline{\mathcal{N}}^{-\infty}\left(\Omega \times \mathbb{R}^{n}\right), \\
\tilde{\mathcal{S}}_{\mathfrak{B}}^{-\infty}\left(\Omega \times \mathbb{R}^{n}\right) & :=\underline{\mathcal{S}}_{\mathfrak{B}}^{-\infty}\left(\Omega \times \mathbb{R}^{n}\right) / \underline{\mathcal{N}}^{-\infty}\left(\Omega \times \mathbb{R}^{n}\right) .
\end{aligned}
$$

If $\Omega$ is an open subset of $\mathbb{R}^{n}$, then the elements of $\mathcal{S}_{\mathfrak{B}}^{m}\left(\Omega \times \mathbb{R}^{n}\right), \quad \tilde{\mathcal{S}}_{\mathfrak{B}}^{m}\left(\Omega \times \mathbb{R}^{n}\right)$, and $\tilde{\mathcal{S}}_{\mathfrak{B}}^{m,-\infty}\left(\Omega \times \mathbb{R}^{n}\right)$ are called $\mathfrak{B}$-nets of symbols of order $m, \mathfrak{B}$-generalized symbols of order $m$, and $\mathfrak{B}$-generalized symbols of refined order $m$, respectively. The sets $\underline{\mathcal{S}}_{\mathfrak{B}}^{-\infty}\left(\Omega \times \mathbb{R}^{n}\right)$ and $\tilde{\mathcal{S}}_{\mathfrak{B}}^{-\infty}\left(\Omega \times \mathbb{R}^{n}\right)$ constitute the $\mathfrak{B}$-nets of smoothing symbols and the $\mathfrak{B}$-generalized smoothing symbols, respectively. Finally, if instead of $\Omega$ we have $\Omega \times \Omega$, then we use the notion of amplitude rather than of symbol. 
As mentioned in [7], if we require the above symbol estimates to hold only for small values of $\varepsilon$, then this results in larger spaces of nets of symbols as well as somewhat larger quotient spaces. Even though it is then possible to define pseudodifferential operators with $\mathfrak{B}$-generalized symbols equally well, we prefer to consider here the spaces $\tilde{\mathcal{S}}_{\mathfrak{B}}^{m}\left(\Omega \times \mathbb{R}^{n}\right)$ defined above, since a complete pseudodifferential calculus can be developed.

Remark A 2. Note that, as special cases of $\mathfrak{B}$-generalized symbols, we obtain for $\mathfrak{B}=\Pi_{\text {sc }}$ the slow-scale generalized symbols, and for $\mathfrak{B}=\left\{\left(\varepsilon^{-N}\right)_{\varepsilon}: N \in \mathbb{N}\right\}$ the regular generalized symbols $\tilde{\mathcal{S}}_{\mathrm{rg}}^{m}\left(\Omega \times \mathbb{R}^{n}\right)$ introduced in earlier work [7], to which we refer for further details and notations concerning regular symbols and amplitudes.

In the following we say that $\left(a_{\varepsilon}\right)_{\varepsilon} \in \mathcal{S}_{\mathfrak{B}}^{m}\left(\Omega \times \mathbb{R}^{n}\right)$ is of growth type $\left(\omega_{\varepsilon}\right)_{\varepsilon} \in \mathfrak{B}$ on $K \Subset \Omega$ if $\omega_{\varepsilon}$ is an upper bound for each seminorm $\left|a_{\varepsilon}\right|_{K, \alpha, \beta}^{(m)}$. We list the basic steps in establishing a calculus with asymptotic expansions.

Definition A 3. Let $\left\{m_{j}\right\}_{j}$ be a decreasing sequence of real numbers tending to $-\infty$ and let $\left\{\left(a_{j, \varepsilon}\right)_{\varepsilon}\right\}_{j}$ be a sequence of $\mathfrak{B}$-nets of symbols $\left(a_{j, \varepsilon}\right)_{\varepsilon} \in \mathcal{S}_{\mathfrak{B}^{\prime}}^{m_{j}}\left(\Omega \times \mathbb{R}^{n}\right)$ such that

$$
\forall K \Subset \Omega \exists\left(\omega_{\varepsilon}\right)_{\varepsilon} \in \mathfrak{B} \forall j \in \mathbb{N}:\left(a_{j, \varepsilon}\right)_{\varepsilon} \text { is of growth type }\left(\omega_{\varepsilon}\right)_{\varepsilon} \text { on } K .
$$

We say that $\sum_{j}\left(a_{\varepsilon}\right)_{\varepsilon}$ is the asymptotic expansion of $\left(a_{\varepsilon}\right)_{\varepsilon} \in \mathcal{E}\left[\Omega \times \mathbb{R}^{n}\right],\left(a_{\varepsilon}\right)_{\varepsilon} \sim \sum_{j}\left(a_{j, \varepsilon}\right)_{\varepsilon}$ for short, if and only if, for all $K \Subset \Omega$, there exists $\left(\omega_{\varepsilon}\right)_{\varepsilon} \in \mathfrak{B}$ such that, for all $r \geqslant 1$, the difference

$$
\left(a_{\varepsilon}-\sum_{j=0}^{r-1} a_{j, \varepsilon}\right)_{\varepsilon}
$$

belongs to $\underline{\mathcal{S}}_{\mathfrak{B}}^{m_{r}}\left(\Omega \times \mathbb{R}^{n}\right)$ and is of growth type $\left(\omega_{\varepsilon}\right)_{\varepsilon}$ on $K$.

Theorem A 4. For any sequence of $\mathfrak{B}$-nets of symbols $\left(a_{j, \varepsilon}\right)_{\varepsilon} \in \mathcal{S}_{\mathfrak{B}}^{m_{j}}\left(\Omega \times \mathbb{R}^{n}\right)$ as in Definition A 3, there exists $\left(a_{\varepsilon}\right)_{\varepsilon} \in \mathcal{S}_{\mathfrak{B}}^{m_{0}}\left(\Omega \times \mathbb{R}^{n}\right)$ such that $\left(a_{\varepsilon}\right)_{\varepsilon} \sim \sum_{j}\left(a_{j, \varepsilon}\right)_{\varepsilon}$. Moreover, if $\left(a_{\varepsilon}^{\prime}\right)_{\varepsilon} \sim \sum_{j}\left(a_{j, \varepsilon}\right)_{\varepsilon}$, then $\left(a_{\varepsilon}-a_{\varepsilon}^{\prime}\right) \in \mathcal{S}_{\mathfrak{B}}^{-\infty}\left(\Omega \times \mathbb{R}^{n}\right)$.

This result is easily obtained from the proof of [7, Theorem 5.3], and noting that we do not have powers of $\varepsilon$ depending on $x$-derivatives and replacing $\varepsilon^{-N}$ by $\left(\omega_{\varepsilon}\right)_{\varepsilon} \in$ $\mathfrak{B}$. The following proposition concerning negligible nets of symbols is a consequence of $[\mathbf{7}$, Theorem 5.4].

Proposition A 5. Let $\left\{m_{j}\right\}_{j}$ be a decreasing sequence of real numbers tending to $-\infty$ and let $\left(a_{j, \varepsilon}\right)_{\varepsilon} \in \underline{\mathcal{N}}^{m_{j}}\left(\Omega \times \mathbb{R}^{n}\right)$ for all $j$. Then there exists $\left(a_{\varepsilon}\right)_{\varepsilon} \in \underline{\mathcal{N}}^{m_{0}}\left(\Omega \times \mathbb{R}^{n}\right)$ such that, for all $r \geqslant 1$, we have

$$
\left(a_{\varepsilon}-\sum_{j=0}^{r-1} a_{j, \varepsilon}\right)_{\varepsilon} \in \underline{\mathcal{N}}^{m_{r}}\left(\Omega \times \mathbb{R}^{n}\right) .
$$

Definition A 6. Let $\left\{m_{j}\right\}_{j}$ be a decreasing sequence of real numbers tending to $-\infty$. Let $\left\{a_{j}\right\}_{j}$ be a sequence of $\mathfrak{B}$-generalized symbols $a_{j} \in \tilde{\mathcal{S}}_{\mathfrak{B}}^{m_{j}}\left(\Omega \times \mathbb{R}^{n}\right)$ such that there exists a choice of representatives $\left(a_{j, \varepsilon}\right)_{\varepsilon}$ of $a_{j}$ satisfying (A 1$)$. We say that $\sum_{j} a_{j}$ is 
the asymptotic expansion of $a \in \tilde{\mathcal{S}}_{\mathfrak{B}}^{m_{0}}\left(\Omega \times \mathbb{R}^{n}\right), a \sim \sum_{j} a_{j}$ for short, if and only if there exists a representative $\left(a_{\varepsilon}\right)_{\varepsilon}$ of $a$ and for all $j$ representatives $\left(a_{j, \varepsilon}\right)_{\varepsilon}$ of $a_{j}$, such that $\left(a_{\varepsilon}\right)_{\varepsilon} \sim \sum_{j}\left(a_{j, \varepsilon}\right)_{\varepsilon}$.

Proposition A 5 allows us to claim that $a \sim \sum_{j} a_{j}$ if and only if, for any choice of representatives $\left(a_{j, \varepsilon}\right)_{\varepsilon}$ of $a_{j}$, there exists a representative $\left(a_{\varepsilon}\right)_{\varepsilon}$ of $a$ such that $\left(a_{\varepsilon}\right)_{\varepsilon} \sim \sum\left(a_{j, \varepsilon}\right)_{\varepsilon}$. This observation, combined with Theorem A 4, is crucial for the proof of Theorem A 7 .

Theorem A 7. For any sequence of $\mathfrak{B}$-generalized symbols $a_{j} \in \tilde{\mathcal{S}}_{\mathfrak{B}}^{m_{j}}\left(\Omega \times \mathbb{R}^{n}\right)$ as in Definition A6, there exists $a \in \tilde{\mathcal{S}}_{\mathfrak{B}}^{m_{0}}\left(\Omega \times \mathbb{R}^{n}\right)$ such that $a \sim \sum_{j} a_{j}$. Moreover, if $b \in \tilde{\mathcal{S}}_{\mathfrak{B}}^{m_{0}}\left(\Omega \times \mathbb{R}^{n}\right)$ has asymptotic expansion $\sum_{j} a_{j}$, then there exists a representative $\left(a_{\varepsilon}\right)_{\varepsilon}$ of $a$ and a representative $\left(b_{\varepsilon}\right)_{\varepsilon}$ of $b$ such that

$$
\left(a_{\varepsilon}-b_{\varepsilon}\right)_{\varepsilon} \in \underline{\mathcal{S}}_{\mathfrak{B}}^{-\infty}\left(\Omega \times \mathbb{R}^{n}\right) .
$$

Remark A 8. Definition A 6 can be stated for $\mathfrak{B}$-generalized symbols of refined order. More precisely, if $\left\{m_{j}\right\}_{j}$ is a sequence as above,

$$
a \in \tilde{\mathcal{S}}_{\mathfrak{B}}^{m_{0},-\infty}\left(\Omega \times \mathbb{R}^{n}\right), \quad a_{j} \in \tilde{\mathcal{S}}_{\mathfrak{B}}^{m_{j},-\infty}\left(\Omega \times \mathbb{R}^{n}\right) \text { for all } j
$$

and $\left(a_{\varepsilon}\right)_{\varepsilon}$ and $\left(a_{j, \varepsilon}\right)_{\varepsilon}$ denote representatives of $a$ and $a_{j}$, respectively, the following assertions are equivalent:

(1) $a \sim \sum_{j} a_{j}$,

(2) $\exists\left(a_{\varepsilon}\right)_{\varepsilon} \exists\left\{\left(a_{j, \varepsilon}\right)_{\varepsilon}\right\}_{j}:\left(a_{\varepsilon}\right)_{\varepsilon} \sim \sum_{j}\left(a_{j, \varepsilon}\right)_{\varepsilon}$,

(3) $\forall\left\{\left(a_{j, \varepsilon}\right)_{\varepsilon}\right\}_{j} \exists\left(a_{\varepsilon}\right)_{\varepsilon}:\left(a_{\varepsilon}\right)_{\varepsilon} \sim \sum_{j}\left(a_{j, \varepsilon}\right)_{\varepsilon}$,

(4) $\forall\left\{\left(a_{j, \varepsilon}\right)_{\varepsilon}\right\}_{j} \forall\left(a_{\varepsilon}\right)_{\varepsilon}:\left(a_{\varepsilon}\right)_{\varepsilon} \sim \sum_{j}\left(a_{j, \varepsilon}\right)_{\varepsilon}$.

We briefly recall the main definitions and results concerning pseudodifferential operators with $\mathfrak{B}$-generalized symbols. As already observed after Theorem A 4 , the proofs are obtained from the corresponding ones in [7], with the slight difference of having a net $\left(\omega_{\varepsilon}\right)_{\varepsilon}$ in $\mathfrak{B}$ instead of a power $\varepsilon^{-N}$.

Definition A 9. Let $a \in \tilde{\mathcal{S}}_{\mathfrak{B}}^{m}\left(\Omega \times \mathbb{R}^{n}\right)$. The pseudodifferential operator with $\mathfrak{B}$ generalized symbol $a$ is the map $a(x, D): \mathcal{G}_{\mathrm{c}}(\Omega) \rightarrow \mathcal{G}(\Omega)$ given by the formula

$$
a(x, D) u(x):=\int_{\mathbb{R}^{n}} \mathrm{e}^{\mathrm{i} x \xi} a(x, \xi) \hat{u}(\xi) \mathrm{d} \xi:=\left[\left(\int_{\mathbb{R}^{n}} \mathrm{e}^{\mathrm{i} x \xi} a_{\varepsilon}(x, \xi) \hat{u}_{\varepsilon}(\xi) \mathrm{d} \xi\right)_{\varepsilon}\right] .
$$

Proposition 4.7 in [7] guarantees the well-definedness of $a(x, D)$ as well as the additional mapping property $a(x, D): \mathcal{G}_{\mathrm{c}}^{\infty}(\Omega) \rightarrow \mathcal{G}^{\infty}(\Omega)$.

In the following, we make occasional use of some basic properties of the space $L\left(\mathcal{G}_{\mathrm{c}}(\Omega), \widetilde{\mathbb{C}}\right)$ of $\widetilde{\mathbb{C}}$-linear maps from $\mathcal{G}_{\mathrm{c}}(\Omega)$ to $\widetilde{\mathbb{C}}$. In particular, we recall that $\mathcal{G}(\Omega)$ is linearly embedded into $L\left(\mathcal{G}_{\mathrm{c}}(\Omega), \widetilde{\mathbb{C}}\right)$ via generalized integration and $L\left(\mathcal{G}_{\mathrm{c}}(\Omega), \widetilde{\mathbb{C}}\right)$ is a sheaf with respect to $\Omega$. This and further results are discussed in detail in $[\mathbf{7}, \S 2]$. 
Definition A 10. Let $a \in \tilde{\mathcal{S}}_{\mathfrak{B}}^{m}\left(\Omega \times \mathbb{R}^{n}\right)$. The kernel of $a(x, D)$ is the $\widetilde{\mathbb{C}}$-linear map $k: \mathcal{G}_{\mathrm{c}}(\Omega \times \Omega) \rightarrow \widetilde{\mathbb{C}}$ defined by

$$
k(u):=\int_{\Omega} a(x, D)(u(x, \cdot))(x) \mathrm{d} x .
$$

To see that formula (A 2) makes sense for $k$ as an element of $L\left(\mathcal{G}_{\mathrm{c}}(\Omega \times \Omega), \widetilde{\mathbb{C}}\right)$, we may reason as in [7, Proposition 3.10 and Remark 3.11] that we have $a(x, D)(u(x, \cdot)) \in \mathcal{G}_{\mathrm{c}}(\Omega)$. Moreover, for all $u, v \in \mathcal{G}_{\mathrm{c}}(\Omega)$,

$$
k(v \otimes u)=\int_{\Omega} a(x, D) u(x) v(x) \mathrm{d} x=\int_{\Omega} u(x)^{\mathrm{T}} a(x, D) v(x) \mathrm{d} x,
$$

where $v \otimes u:=\left[\left(v_{\varepsilon}(x) u_{\varepsilon}(y)\right)_{\varepsilon}\right] \in \mathcal{G}_{\mathrm{c}}(\Omega \times \Omega)$; as a consequence, since $\mathcal{G}(\Omega)$ is embedded into $L\left(\mathcal{G}_{\mathrm{c}}(\Omega), \widetilde{\mathbb{C}}\right)$, pseudodifferential operators having the same kernel are identical.

We say that a pseudodifferential operator with a $\mathfrak{B}$-generalized symbol is properly supported if the support of its kernel is a proper set of $\Omega \times \Omega$. As shown in [7, Proposition 4.17], we find that any properly supported pseudodifferential operator $a(x, D)$ maps $\mathcal{G}_{\mathrm{c}}(\Omega)$ into $\mathcal{G}_{\mathrm{c}}(\Omega), \mathcal{G}_{\mathrm{c}}^{\infty}(\Omega)$ into $\mathcal{G}_{\mathrm{c}}^{\infty}(\Omega)$ and can be extended uniquely to a linear map from $\mathcal{G}(\Omega)$ into $\mathcal{G}(\Omega)$ such that, for all $u \in \mathcal{G}(\Omega)$ and $v \in \mathcal{G}_{\mathrm{c}}(\Omega)$,

$$
\int_{\Omega} a(x, D) u(x) v(x) \mathrm{d} x=\int_{\Omega} u(x)^{\mathrm{T}} a(x, D) v(x) \mathrm{d} x .
$$

This extension maps $\mathcal{G}^{\infty}(\Omega)$ into $\mathcal{G}^{\infty}(\Omega)$. By the same reasoning as in [7, Proposition 4.11], we prove that each pseudodifferential operator $a(x, D)$ with $\mathfrak{B}$-generalized symbol has the pseudolocality property, i.e.

$$
\operatorname{singsupp}_{g}(a(x, D) u) \subseteq \operatorname{singsupp}_{g}(u)
$$

for all $u \in \mathcal{G}_{\mathrm{c}}(\Omega)$, and that this result is valid for all $u$ in $\mathcal{G}(\Omega)$ if $a(x, D)$ is properly supported.

Pseudodifferential operators can also be defined by $\mathfrak{B}$-generalized amplitudes. This means that $b \in \tilde{\mathcal{S}}_{\mathfrak{B}}^{m}\left(\Omega \times \Omega \times \mathbb{R}^{n}\right)$ defines the action of the corresponding operator on $u \in \mathcal{G}_{\mathrm{c}}(\Omega)$ via the oscillatory integral

$$
B u(x):=\int_{\Omega \times \mathbb{R}^{n}} \mathrm{e}^{\mathrm{i}(x-y) \xi} b(x, y, \xi) u(y) \mathrm{d} y \mathrm{~d} \xi,
$$

which gives a Colombeau function in $\mathcal{G}(\Omega)[\mathbf{7}, \S 3]$. It is clear that the same constructions concerning kernel and properly supported pseudodifferential operators are still valid. For the sake of completeness we recall that any integral operator $R$ with regular kernel, i.e. any operator of the form

$$
R u(x)=\int_{\Omega} k(x, y) u(y) \mathrm{d} y, \quad u \in \mathcal{G}_{\mathrm{c}}(\Omega),
$$

where $k \in \mathcal{G}^{\infty}(\Omega \times \Omega)$ can be written as a pseudodifferential operator with regular amplitude in $\widetilde{\mathcal{S}}_{\mathrm{rg}}^{-\infty}\left(\Omega \times \Omega \times \mathbb{R}^{n}\right)$ and vice versa if $B$ is a pseudodifferential operator with amplitude in

$$
\tilde{\mathcal{S}}_{\mathfrak{B}}^{-\infty}\left(\Omega \times \Omega \times \mathbb{R}^{n}\right)
$$


then its kernel is a regular generalized function. Finally, an operator with regular kernel is regularizing, i.e. it maps $\mathcal{G}_{\mathrm{c}}(\Omega)$ into $\mathcal{G}^{\infty}(\Omega)$.

Consider $a \in \tilde{\mathcal{S}}_{\mathfrak{B}}^{m}\left(\Omega \times \mathbb{R}^{n}\right)$, where $k$ is the kernel of $a(x, D)$ and let $\chi \in \mathcal{C}^{\infty}(\Omega \times \Omega)$ be a proper function identically 1 in a neighbourhood of $\operatorname{supp} k$. We may write $a(x, D)=$ $a_{0}(x, D)+a_{1}(x, D)$, where

$$
a_{0}(x, D) u(x):=\int_{\Omega \times \mathbb{R}^{n}} \mathrm{e}^{\mathrm{i}(x-y) \xi} a(x, \xi) \chi(x, y) u(y) \mathrm{d} y \mathrm{~d} \xi
$$

is a properly supported pseudodifferential operator with generalized amplitude

$$
a(x, \xi) \chi(x, y) \in \tilde{\mathcal{S}}_{\mathfrak{B}}^{m}\left(\Omega \times \Omega \times \mathbb{R}^{n}\right)
$$

and

$$
a_{1}(x, D) u(x):=\int_{\Omega \times \mathbb{R}^{n}} \mathrm{e}^{\mathrm{i}(x-y) \xi} a(x, \xi)(1-\chi(x, y)) u(y) \mathrm{d} y \mathrm{~d} \xi
$$

is an operator with regular kernel in $\mathcal{G}^{\infty}(\Omega \times \Omega)$. The following theorem shows that every properly supported pseudodifferential operator defined via an amplitude can be written in the form of Definition A 9. This is the main tool in the proof of Theorem A 12.

Theorem A 11. For any properly supported pseudodifferential operator $A$ with amplitude $a \in \tilde{\mathcal{S}}_{\mathfrak{B}}^{m}\left(\Omega \times \Omega \times \mathbb{R}^{n}\right)$, there exists $\sigma \in \tilde{\mathcal{S}}_{\mathfrak{B}}^{m}\left(\Omega \times \mathbb{R}^{n}\right)$ such that $A \equiv \sigma(x, D)$ on $\mathcal{G}_{\mathrm{c}}(\Omega)$ and

$$
\left.\sigma \sim \sum_{\gamma} \frac{1}{\gamma !} \partial_{\xi}^{\gamma} D_{y}^{\gamma} a(x, y, \xi)\right|_{x=y}
$$

Theorem A 12. Let $a \in \tilde{\mathcal{S}}_{\mathfrak{B}}^{m}\left(\Omega \times \mathbb{R}^{n}\right)$ and $b \in \tilde{\mathcal{S}}_{\mathfrak{B}}^{m^{\prime}}\left(\Omega \times \mathbb{R}^{n}\right)$ be $\mathfrak{B}$-generalized symbols. If the corresponding pseudodifferential operators are properly supported, then

(i) there exists $a^{\prime} \in \tilde{\mathcal{S}}_{\mathfrak{B}}^{m}\left(\Omega \times \mathbb{R}^{n}\right)$ such that ${ }^{\mathrm{T}} a(x, D) \equiv a^{\prime}(x, D)$ on $\mathcal{G}_{\mathrm{c}}(\Omega)$ and

$$
a^{\prime} \sim \sum_{\gamma} \frac{(-1)^{|\gamma|}}{\gamma !} \partial_{\xi}^{\gamma} D_{x}^{\gamma} a(x,-\xi)
$$

(ii) there exists $a^{*} \in \tilde{\mathcal{S}}_{\mathfrak{B}}^{m}\left(\Omega \times \mathbb{R}^{n}\right)$ such that $a(x, D)^{*} \equiv a^{*}(x, D)$ on $\mathcal{G}_{\mathrm{c}}(\Omega)$ and

$$
a^{*} \sim \sum_{\gamma} \frac{1}{\gamma !} \partial_{\xi}^{\gamma} D_{x}^{\gamma} \bar{a}
$$

(iii) there exists $a \sharp b \in \tilde{\mathcal{S}}_{\mathfrak{B}}^{m+m^{\prime}}\left(\Omega \times \mathbb{R}^{n}\right)$ such that $a(x, D) \circ b(x, D) \equiv a \sharp b(x, D)$ on $\mathcal{G}_{\mathrm{c}}(\Omega)$ and

$$
a \sharp b \sim \sum_{\gamma} \frac{1}{\gamma !} \partial_{\xi}^{\gamma} a D_{x}^{\gamma} b .
$$


Proof. We briefly sketch the proof of assertion (ii). For the details concerning the transposed operator and the product we refer to [7]. By definition of the formal adjoint, $\langle a(x, D) v, \bar{u}\rangle=\langle v, \overline{a(x, D) * u}\rangle$ for all $u, v \in \mathcal{G}_{\mathrm{c}}(\Omega)$. This means

$$
\left\langle v, \overline{a(x, D)^{*} u}\right\rangle=\int_{\Omega} \overline{\int_{\Omega \times \mathbb{R}^{n}} \mathrm{e}^{\mathrm{i}(x-y) \xi} \bar{a}(y, \xi) u(y) \mathrm{d} y \mathrm{~d} \xi} v(x) \mathrm{d} x,
$$

which, from the embedding of $\mathcal{G}(\Omega)$ into $L\left(\mathcal{G}_{\mathrm{c}}(\Omega), \widetilde{\mathbb{C}}\right)$, leads us to

$$
a(x, D)^{*} u=\int_{\Omega \times \mathbb{R}^{n}} \mathrm{e}^{\mathrm{i}(x-y) \xi} \bar{a}(y, \xi) u(y) \mathrm{d} y \mathrm{~d} \xi
$$

Now, $a(x, D)^{*}$ is a properly supported pseudodifferential operator with amplitude $\bar{a}(y, \xi) \in \tilde{\mathcal{S}}_{\mathfrak{B}}^{m}\left(\Omega \times \Omega \times \mathbb{R}^{n}\right)$, and an application of Theorem A 11 completes the proof.

Along the lines of [7, Proposition 5.17] we easily prove that the composition of a properly supported pseudodifferential operator with $\mathfrak{B}$-generalized symbol and an operator with regular kernel is an operator with regular kernel. Therefore, combining (A 3) and (A 4) with Theorem A 12, we find that, for arbitrary pseudodifferential operators with $\mathfrak{B}$-generalized symbol, the equalities (i) and (ii) on $\mathcal{G}_{\mathrm{c}}(\Omega)$ are valid modulo some operator with regular kernel. Furthermore, the composition $a(x, D) \circ b(x, D)$, where at least one of the operators is properly supported, is a pseudodifferential operator $a \sharp b(x, D)$ modulo some operator with regular kernel.

\section{Remark A 13.}

(i) It is clear from the structure of $\tilde{\mathcal{S}}_{\mathfrak{B}}^{m,-\infty}\left(\Omega \times \mathbb{R}^{n}\right)$ that all the definitions and results of this appendix can be stated for symbols of refined order.

(ii) Let now $\mathcal{S}_{\rho, \delta}^{m}\left[\Omega \times \mathbb{R}^{n}\right]$ be the set of all nets $\left(a_{\varepsilon}\right)_{\varepsilon} \in S_{\rho, \delta}^{m}\left(\Omega \times \mathbb{R}^{n}\right)^{(0,1]}$ with

$$
\left|a_{\varepsilon}\right|_{K, \alpha, \beta, \rho, \delta}^{(m)}:=\sup _{x \in K, \xi \in \mathbb{R}^{n}}\left|\partial_{\xi}^{\alpha} \partial_{x}^{\beta} a_{\varepsilon}(x, \xi)\right|\langle\xi\rangle^{-m+\rho|\alpha|-\delta|\beta|}
$$

seminorm in $S_{\rho, \delta}^{m}\left(\Omega \times \mathbb{R}^{n}\right)$. We define $\underline{\mathcal{S}}_{\mathfrak{B}, \rho, \delta}^{m}\left(\Omega \times \mathbb{R}^{n}\right)$ and $\underline{\mathcal{N}}_{\rho, \delta}^{m}\left(\Omega \times \mathbb{R}^{n}\right)$ as the subspaces of $\mathcal{S}_{\rho, \delta}^{m}\left[\Omega \times \mathbb{R}^{n}\right]$ obtained by requiring the same estimate of $\left|a_{\varepsilon}\right|_{K, \alpha, \beta, \rho, \delta}^{(m)}$ as in $\mathcal{S}_{\mathfrak{B}}^{m}\left(\Omega \times \mathbb{R}^{n}\right)$ and $\underline{\mathcal{N}}^{m}\left(\Omega \times \mathbb{R}^{n}\right)$, respectively. In conclusion, under the assumption $0 \leqslant \delta<\rho \leqslant 1$ it is possible to develop a pseudodifferential calculus for generalized symbols in

$$
\tilde{\mathcal{S}}_{\mathfrak{B}, \rho, \delta}^{m}\left(\Omega \times \mathbb{R}^{n}\right):=\underline{\mathcal{S}}_{\mathfrak{B}, \rho, \delta}^{m}\left(\Omega \times \mathbb{R}^{n}\right) / \underline{\mathcal{N}}_{\rho, \delta}^{m}\left(\Omega \times \mathbb{R}^{n}\right)
$$

and

$$
\tilde{\mathcal{S}}_{\mathfrak{B}, \rho, \delta}^{m,-\infty}\left(\Omega \times \mathbb{R}^{n}\right):=\underline{\mathcal{S}}_{\mathfrak{B}, \rho, \delta}^{m}\left(\Omega \times \mathbb{R}^{n}\right) / \underline{\mathcal{N}}^{-\infty}\left(\Omega \times \mathbb{R}^{n}\right),
$$

as in the classical theory. 
Acknowledgements. G.H. was supported by FWF grant P14576-MAT at the Institut für Technische Mathematik, Geometrie und Bauniformatik, Universität Innsbruck, Austria.

The authors are grateful to the members of DIANA (see www.mat.univie.ac.at/ diana) for several inspiring discussions on the subject. Special thanks go to Roland Steinbauer for having posed the striking question leading the authors to the additional characterization theorem (Theorem 3.11).

\section{References}

1. H. Biagioni and M. Oberguggenberger, Generalized solutions to the KortewegDe Vries and the regularized long-wave equations, SIAM J. Math. Analysis 23 (1992), 923-940.

2. J. F. Colombeau, New generalized functions and multiplication of distributions (NorthHolland, Amsterdam, 1984).

3. J. F. Colombeau, Elementary introduction to new generalized functions (North-Holland, Amsterdam, 1985).

4. N. Dapić, S. Pilipović And D. Scarpalézos, Microlocal analysis of Colombeau's generalized functions: propagation of singularities, J. Analyse Math. 75 (1998), 51-66.

5. G. B. Folland, Introduction to partial differential equations, 2nd edn (Princeton University Press, Princeton, NJ, 1995).

6. C. Garetto, Pseudodifferential operators in algebras of generalized functions and global hypoellipticity, Acta Appl. Math. 82 (2004), 123-174.

7. C. Garetto, T. Gramchev and M. Oberguggenberger, Pseudodifferential operators with generalized symbols and regularity theory, Preprint, University of Innsbruck (2003).

8. M. Grosser, M. Kunzinger, M. Oberguggenberger and R. Steinbauer, Geometric theory of generalized functions, Mathematics and its Applications, vol. 537 (Kluwer, Dordrecht, 2001).

9. L. Hörmander, Fourier integral operators, I, Acta Math. 127 (1971), 79-183.

10. L. HÖRMANDER, The analysis of linear partial differential operators, vol. III (Springer, 1985).

11. G. Hörmann, Integration and microlocal analysis in Colombeau algebras, J. Math. Analysis Applic. 239 (1999), 332-348.

12. G. Hörmann, First-order hyperbolic pseudodifferential equations with generalized symbols, J. Math. Analysis Applic. 293 (2004), 40-56.

13. G. Hörmann And M. V. DE Hoop, Microlocal analysis and global solutions of some hyperbolic equations with discontinuous coefficients, Acta Appl. Math. 67 (2001), 173224.

14. G. Hörmann And M. Kunzinger, Microlocal analysis of basic operations in Colombeau algebras, J. Math. Analysis Applic. 261 (2001), 254-270.

15. G. Hörmann AND M. OBERGUGGENBERGER, Elliptic regularity and solvability for partial differential equations with Colombeau coefficients, Electron. J. Diff. Eqns 2004 (2004), $1-30$.

16. G. Hörmann, M. Oberguggenberger and S. Pilipovic, Microlocal hypoellipticity of linear partial differential operators with generalized functions as coefficients, Trans. Am. Math. Soc., in press.

17. F. Lafon and M. Oberguggenberger, Generalized solutions to symmetric hyperbolic systems with discontinuous coefficients: the multidimensional case, J. Math. Analysis Applic. 160 (1991), 93-106. 
18. M. NedelJKov And S. Pilipović, Hypoelliptic differential operators with generalized constant coefficients, Proc. Edinb. Math. Soc. 41 (1998), 47-60.

19. M. Nedeljkov, S. Pilipović And D. Scarpalézos, The linear theory of Colombeau generalized functions, Pitman Research Notes in Mathematics, vol. 385 (Longman Scientific \& Technical, Harlow, 1998).

20. M. Oberguggenberger, Multiplication of distributions and applications to partial differential equations, Pitman Research Notes in Mathematics, vol. 259 (Longman Scientific \& Technical, Harlow, 1992). 